\title{
Estimation of the Basic Reproductive Ratio for Dengue Fever at the Take-Off Period of Dengue Infection
}

\author{
Jafaruddin, ${ }^{1,2}$ Sapto W. Indratno, ${ }^{1}$ Nuning Nuraini, ${ }^{1}$ Asep K. Supriatna, ${ }^{3}$ and Edy Soewono ${ }^{1}$ \\ ${ }^{1}$ Departemen Matematika, FMIPA, Institut Teknologi Bandung, Bandung, Indonesia \\ ${ }^{2} J u r u s a n$ Matematika, FST, Universitas Nusa Cendana, Kupang, Indonesia \\ ${ }^{3}$ Jurusan Matematika, FMIPA, Universitas Padjadjaran, Bandung, Indonesia
}

Correspondence should be addressed to Jafaruddin; jafaruddin.hamid@yahoo.com

Received 22 December 2014; Revised 6 July 2015; Accepted 7 July 2015

Academic Editor: Chung-Min Liao

Copyright (C) 2015 Jafaruddin et al. This is an open access article distributed under the Creative Commons Attribution License, which permits unrestricted use, distribution, and reproduction in any medium, provided the original work is properly cited.

\begin{abstract}
Estimating the basic reproductive ratio $\mathscr{R}_{0}$ of dengue fever has continued to be an ever-increasing challenge among epidemiologists. In this paper we propose two different constructions to estimate $\mathscr{R}_{0}$ which is derived from a dynamical system of host-vector dengue transmission model. The construction is based on the original assumption that in the early states of an epidemic the infected human compartment increases exponentially at the same rate as the infected mosquito compartment (previous work). In the first proposed construction, we modify previous works by assuming that the rates of infection for mosquito and human compartments might be different. In the second construction, we add an improvement by including more realistic conditions in which the dynamics of an infected human compartments are intervened by the dynamics of an infected mosquito compartment, and vice versa. We apply our construction to the real dengue epidemic data from SB Hospital, Bandung, Indonesia, during the period of outbreak Nov. 25, 2008-Dec. 2012. We also propose two scenarios to determine the take-off rate of infection at the beginning of a dengue epidemic for construction of the estimates of $\mathscr{R}_{0}$ : scenario I from equation of new cases of dengue with respect to time (daily) and scenario II from equation of new cases of dengue with respect to cumulative number of new cases of dengue. The results show that our first construction of $\mathscr{R}_{0}$ accommodates the take-off rate differences between mosquitoes and humans. Our second construction of the $\mathscr{R}_{0}$ estimation takes into account the presence of infective mosquitoes in the early growth rate of infective humans and vice versa. We conclude that the second approach is more realistic, compared with our first approach and the previous work.
\end{abstract}

\section{Introduction}

Dengue is a mosquito-borne viral disease found in more than 100 countries around the world which are mostly located in tropical and subtropical countries. This disease is transmitted through the bites of female Aedes aegypti. In recent years, dengue transmission has increased predominantly in urban and semiurban areas and has become a major international public health concern. Controlling of dengue fever has been conducted continuously, but the spread of dengue virus is still increasing in many countries. Many efforts have been conducted to control the spread of the virus, for instance, a reduction in the population of Aedes aegypti in the field [1]. Fumigation methods have been used to reduce mosquitoes, and the use of temephos has been utilized to reduce the larvae. Until recently, there was no vaccine against any of the four virus serotypes (DEN-1, DEN-2, DEN-3, and DEN-4). To cure patients, treatment in hospitals is usually given in the form of supportive care, which includes bed rest, antipyretics, and analgesics [2]. Mathematical models have proved to be useful tools in the understanding of dengue transmission [35]. So far the dynamics of the dengue transmission are still an interesting issue in epidemiological modeling.

The first model of dengue transmission and the stability analysis of equilibrium points are shown in [6] in which the basic reproductive ratio $\mathscr{R}_{0}$ is constructed from the stability condition of the disease-free equilibrium. The general concept of $\mathscr{R}_{0}$ can be seen in $[7,8]$ which was adapted to various infectious disease models $[9,10]$. The difficulty in using $\mathscr{R}_{0}$ for measuring the level of dengue endemicity in the field is that $\mathscr{R}_{0}$ often depends on several parameters (biological, environmental, transmission, etc), which are difficult to be 
obtained from the field. With limited information about mosquitos such as the mosquito population size the estimate of $\mathscr{R}_{0}$ can not be computed from the dynamical model only. With daily human incidence being the only available data, it is natural to ask how to extract relevant parameters from the data to fit in the model. A simple approach has been achieved by assuming that both a human and mosquito undergo linear growth at the early state of infection. As shown in [11-14] the estimation does not distinguish between the growth rate of an infected human and the growth rate of infected mosquitoes and does not yet consider how the interaction between infected mosquitoes and infected humans influences the early growth of a dengue epidemic. There are other problems that are treated in a recent paper [15] which examines two models vector-borne infections, namely, dengue transmission. In this paper, we construct $\mathscr{R}_{0}$ estimation by taking into account the difference between the growth rate of an infected human and infected mosquito and the effect of the interaction between them and the characteristics of dengue incidence data.

The existing data do not specify the infection status (age of infection) of each patient. Hence we assume that people who come to the hospital should be identified as dengue patient at the end of incubation period (approximately at the 7th day after contact with infected mosquito). Also, we assume that there are no available alternative hosts as blood sources and there are no death and recovery in the early days of dengue infection. The main purpose of this paper is to construct $\mathscr{R}_{0}$ estimation of the real conditions in the field, in which only the daily incidence data are available. Based on the dengue transmission model in [6], we build two different constructions for $\mathscr{R}_{0}$ estimation and used them for estimating the value of $\mathscr{R}_{0}$ for dengue incidence data between the dates Nov. 25, 2008, and 2012 from SB Hospital, Indonesia.

The organization of the paper is given as follows. In Section 2 a dynamical system of a host-vector transmission model is introduced. Based on this dynamical system we derive the basic reproductive ratio of the model. The constructions of the proposed $\mathscr{R}_{0}$ estimation are presented in Section 3. Formulations of the take-off rate of dengue infection are presented in Section 4. We apply the formula to the real data of dengue incidence from SB Hospital, Bandung, Indonesia. All the numerical results are given in Section 5 along with the insights and interpretation from the numerical results. We provide conclusions in Section 6.

\section{Basic Reproductive Ratio of the Dengue Transmission Model}

Generally, for a vector-borne disease, $\mathscr{R}_{0}$ was understood as the number of persons who would be infected from a single person initially infected by a mosquito $[3,16]$. In the host-vector system, the basic reproductive ratio $\mathscr{R}_{0}$ is defined as the expected number of secondary infections resulting directly from a single infected individual, in a virgin population, during the infection period [7]. The general host-vector dengue transmission model was conducted in [6]. Here, we assume that there are no alternative hosts available, as blood sources $m=0$ for system in [6]. Modification of the dengue

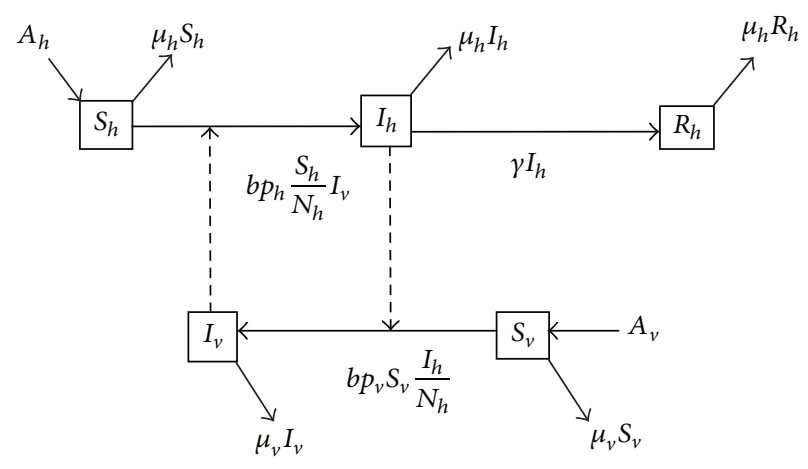

FIGURE 1: The diagram of dengue transmission model.

transmission model in [6], is schematically represented by the diagram in Figure 1, where
$A_{h}:$ recruitment rate of host population;
$N_{h}$ : number of host populations;
$S_{h}$ : susceptible host population size;
$I_{h}$ : infected host population size;
$R_{h}$ : recovered/immunes host population size;
$p_{h}$ : successful transmission probability within host population;
$\mu_{h}$ : birth/death rate of host population;
$\gamma$ : recovery rate of host population;
$A_{v}$ : recruitment rate of vector population;
$N_{v}$ : number of vector populations;
$S_{v}$ : susceptible vector population size;
$I_{\nu}$ : infected vector population size;
$p_{v}$ : transmission probability within the vector popu- lation;
$\mu_{v}$ : death rate of vector population;
$b$ : biting rate of vector population.

The corresponding model of dengue transmission in the short period dengue infection is given in [17] as follows:

$$
\begin{aligned}
& \frac{d S_{h}}{d t}=A_{h}-\frac{b p_{h} S_{h} I_{v}}{N_{h}}-\mu_{h} S_{h}, \\
& \frac{d I_{h}}{d t}=\frac{b p_{h} S_{h} I_{v}}{N_{h}}-\gamma I_{h}-\mu_{h} I_{h}, \\
& \frac{d R_{h}}{d t}=\gamma I_{h}-\mu_{h} R_{h}, \\
& \frac{d S_{v}}{d t}=A_{v}-\frac{b p_{v} S_{v} I_{h}}{N_{h}}-\mu_{v} S_{v}, \\
& \frac{d I_{v}}{d t}=\frac{b p_{v} S_{v} I_{h}}{N_{h}}-\mu_{v} I_{v} .
\end{aligned}
$$

Here, we give a short explanation for system (1) as follows. The effective contact rate to human $b p_{h}$ is the average number 
of contacts per day that would result in infection, if the vector is infectious. Furthermore, the effective contact rate $b p_{v}$ defines the average number of contacts per day that effectively transmit the infection to vectors.

First of all, we determine equilibrium points for the system (1). Because $N_{h}$ and $N_{v}$ are assumed to be constant then from system (1) ones obtain $S_{h}+I_{h}+R_{h}=N_{h}=A_{h} / \mu_{h}$ and $S_{v}+I_{v}=N_{v}=A_{v} / \mu_{v}$. Consequently, we obtain a diseasefree equilibrium point of system (1), which is given by

$$
E_{0}=\left(S_{h 0}=\frac{A_{h}}{\mu_{h}}, I_{h 0}=0, R_{h 0}=0, S_{v 0}=\frac{A_{v}}{\mu_{v}}, I_{v 0}=0\right),
$$

and the endemic equilibrium point in the form of

$$
E_{1}=\left(S_{h}^{*}, I_{h}^{*}, R_{h}^{*}, S_{v}^{*}, I_{v}^{*}\right),
$$

where

$$
\begin{aligned}
& S_{h}^{*}=\frac{\mu_{v}\left(\gamma+\mu_{h}\right) N_{h}^{2}+\mu_{v} N_{h} N_{v}^{2}}{p_{h} \mu_{h} N_{h}+b p_{h} N_{v}}, \\
& I_{h}^{*}=\frac{\mu_{v} \mu_{h} N_{h}\left(\left(b^{2} p_{h} p_{v} / \mu_{v}\left(\gamma+\mu_{h}\right)\right)\left(N_{v} / N_{h}\right)-1\right)}{b p_{v}\left(\mu_{h}+b p_{h} \rho\right)}, \\
& R_{h}^{*}=\frac{\mu_{v} N_{h} \gamma\left(\left(b^{2} p_{h} p_{v} / \mu_{v}\left(\gamma+\mu_{h}\right)\right)\left(N_{v} / N_{h}\right)-1\right)}{b p_{v}\left(\mu_{h}+b p_{h} \rho\right)}, \\
& S_{v}^{*}=\frac{p_{h} \mu_{h} N_{h}+b p_{v} p_{h} N_{v}}{b p_{h}\left(1+b p_{v} \mu_{h}\right)}, \\
& I_{v}^{*} \\
& =\frac{\mu_{v} \mu_{h} N_{h}\left(\gamma+\mu_{h}\right)\left(\left(b^{2} p_{h} p_{v} / \mu_{v}\left(\gamma+\mu_{h}\right)\right)\left(N_{v} / N_{h}\right)-1\right)}{b p_{h}\left(\mu_{v}\left(\gamma+\mu_{h}\right)+\mu_{h} b p_{v}\right)} .
\end{aligned}
$$

Next, we derive the basic reproductive ratio $\mathscr{R}_{0} . \mathscr{R}_{0}$ is often conveniently found using a next generation method $[7,8,18,19]$. We obtain the next generation matrix of system (1), at $E_{0}$, as follows

$$
\mathrm{NGM}=\left[\begin{array}{cc}
0 & \frac{b p_{h}}{\mu_{v}} \\
\frac{b p_{v}}{\left(\gamma+\mu_{h}\right)} \frac{N_{v}}{N_{h}} & 0
\end{array}\right] .
$$

Based on the definition in [20], NGM represents that the number of $b p_{h} / \mu_{v}$ is the generation factor of dengue transmission, from mosquito to human. It means that one mosquito infects $b p_{h}$ humans per unit of time during its infection period $1 / \mu_{v}$, and the number of $\left(b p_{v} /(\gamma+\right.$ $\left.\left.\mu_{h}\right)\right)\left(N_{v} / N_{h}\right)$ is the generation factor of dengue transmission, from human to mosquito. This represents that one human infects $b p_{v}\left(N_{v} / N_{h}\right)$ mosquitoes during one's infection period $1 /\left(\mu_{h}+\gamma\right)$. NGM in (5) have two eigen values, that are $\pm \sqrt{\left(b^{2} p_{h} p_{v} / \mu_{v}\left(\gamma+\mu_{h}\right)\right)\left(N_{v} / N_{h}\right)}$. Therefore the basic reproductive ratio of system (1) is the largest eigen value of NGM, that is

$$
\mathscr{R}_{0}=\sqrt{\frac{b^{2} p_{h} p_{v}}{\mu_{v}\left(\gamma+\mu_{h}\right)} \frac{N_{v}}{N_{h}}} .
$$

It is clear that the endemic equilibrium $E_{1}$ in (3) exists if $\mathscr{R}_{0}>$ 1. In the next section we propose two constructions of an $\mathscr{R}_{0}$ estimation, at the initial growth of dengue infection, based on incidence data.

\section{Construction for $\mathscr{R}_{0}$ Estimation}

Predicting a basic reproductive ratio is not easily done in the field. In the real-life situation, information about the number of mosquitoes, precisely the ratio between mosquito and human population, is limited. Based on the incidence data, we will estimate some related parameters, for the construction of the basic reproductive ratio $\mathscr{R}_{0}$, from dengue incidence data.

This construction is motivated by previous work in [12], which assumes that the infective growth rate is linear at the early state of infection. The exponential growth of the infective compartment, at the early state of infection, is commonly found in the estimation of the basic reproductive ratio. All estimation of $\mathscr{R}_{0}$, in previous works [11, 12, 14, $21,22]$, have been conducted based on the assumption that $K(t) \propto e^{\lambda t}$ varies, where $K(t)$ is the cumulative number of dengue cases, and $\lambda$ is called the force of infection [12, 14]. Chowell et al. [11] called $\lambda$ the initial growth rate of dengue epidemic. Here, we will call $\lambda$ the take-off rate of dengue infection. In the following section, we build two constructions to estimate $\mathscr{R}_{0}$ in (6), which is derived from a dynamical system of the host-vector dengue transmission model in system (1).

First of all we assume the construction of $\mathscr{R}_{0}$ estimation from equation (3) in [12]. The construction of $\mathscr{R}_{0}$ estimation at $E_{0}$ is based on the assumptions that the numbers of infected human population $I_{h}$ and infected mosquito population $I_{v}$ grow exponentially at the same rate at the short time period relative to each other:

$$
\begin{aligned}
& I_{h}(t) \approx I_{h 0} e^{\lambda t}, \\
& I_{v}(t) \approx I_{v 0} e^{\lambda t},
\end{aligned}
$$

where $I_{h 0}$ and $I_{v 0}$ are constant and $\lambda$ is the take-off rate of the initial growth dengue epidemic. Moreover, the number of nonsusceptible hosts and vectors can be assumed to be negligible and by assuming (7) is "like solution" of linearized system (1) for $\mathscr{R}_{0}>1$. These assumptions are also used in $[11,14]$ for calculating the force of infection from the spatial data of a dengue epidemic. Next, by substituting (7) with (1), and assuming that $S_{h} \sim N_{h}$ and $V_{v} \sim N_{V}$ at the early state of an epidemic, we get

$$
\begin{aligned}
\left(\frac{\lambda}{\gamma+\mu_{h}}+1\right) I_{h_{0}} & =\frac{b p_{h}}{\gamma+\mu_{h}} I_{v_{0}}, \\
\left(\frac{\lambda}{\mu_{v}}+1\right) I_{v_{0}} & =\frac{b p_{v}}{\mu_{v}} \frac{N_{v}}{N_{h}} I_{h_{0}} .
\end{aligned}
$$

Multiplying (8) and (9), and using (6), one gets the construction of the $\mathscr{R}_{0}$ estimation as follows:

$$
\begin{aligned}
\mathscr{R}_{0 F}^{2} & =\left(\frac{\lambda}{\mu_{h}+\gamma}+1\right)\left(\frac{\lambda}{\mu_{v}}+1\right)=\frac{b^{2} p_{h} p_{v}}{\mu_{v}\left(\mu_{h}+\gamma\right)} \frac{N_{v}}{N_{h}} \\
& =\mathscr{R}_{0}^{2},
\end{aligned}
$$


which is equivalent to

$$
\mathscr{R}_{0 F}=\sqrt{\frac{\lambda^{2}}{\mu_{v}\left(\mu_{h}+\gamma\right)}+\frac{\left(\mu_{v}+\mu_{h}+\gamma\right) \lambda}{\mu_{v}\left(\mu_{h}+\gamma\right)}+1 .}
$$

The value of $\mathscr{R}_{0 F}$ in (11) is used to estimate $\mathscr{R}_{0}$ in (6), where $\lambda$ is estimated from the dengue incidence data. Note that since $\lambda>0$, then $\mathscr{R}_{0 F}>1$. Note also that a small increase of the value $\lambda$ in the interval $0<\lambda<1$ causes a significant increase of the value $\mathscr{R}_{0 F}$.

Next we introduce our construction of $\mathscr{R}_{0}$ estimation. The first construction is derived under the assumption that, at the beginning of dengue infection, the take-off rate of the host and vector varies differently; that is

$$
\begin{aligned}
& I_{h}(t) \approx I_{h 0} e^{\lambda t}, \\
& I_{v}(t) \approx I_{v 0} e^{k \lambda t},
\end{aligned}
$$

where $k>0$ is the rate of infected mosquitoes per human index. Using the same process as in the derivation of $\mathscr{R}_{0 F}$, one obtains

$$
\begin{aligned}
\mathscr{R}_{0 M F}^{2} & =\left(\frac{\lambda}{\mu_{h}+\gamma}+1\right)\left(\frac{k \lambda}{\mu_{v}}+1\right)=\frac{b^{2} p_{h} p_{v}}{\mu_{v}\left(\mu_{h}+\gamma\right)} \frac{N_{v}}{N_{h}} \\
& =\mathscr{R}_{0}^{2}
\end{aligned}
$$

or, equivalently,

$$
\mathscr{R}_{0 M F}=\sqrt{\frac{k \lambda^{2}}{\mu_{v}\left(\mu_{h}+\gamma\right)}+\frac{\left(\mu_{v}+k\left(\mu_{h}+\gamma\right)\right) \lambda}{\mu_{v}\left(\mu_{h}+\gamma\right)}+1} .
$$

Note that $\mathscr{R}_{0 F}$ is a special case from $\mathscr{R}_{0 M F}$; that is, if $k=1$ then $\mathscr{R}_{0 F}=\mathscr{R}_{0 M F}$. In reality $\mathscr{R}_{0 M F}$ is more realistic than $\mathscr{R}_{0 F}$. From (11) and (14) we obtain

$$
\frac{\mathscr{R}_{0 M F}^{2}}{\mathscr{R}_{0 F}^{2}}=1+\frac{(k-1) \lambda}{\lambda+\mu_{v}} .
$$

The comparison between $\mathscr{R}_{0 M F}$ and $\mathscr{R}_{0 F}$ is shown as the level set of (15) in Figure 2.

Figure 2 shows that the variation in take-off rates might significantly change the values of $\mathscr{R}_{0 F}$ and $\mathscr{R}_{0 M F}$. Both estimations are derived under the assumption that the pure exponential growth phase, of an epidemic, increases or has a fluctuating increase. Accordingly the estimates of $\mathscr{R}_{0}$, obtained from $\mathscr{R}_{0 F}$ and $\mathscr{R}_{0 M F}$, are affected by biasness; that is, the derivations of $\mathscr{R}_{0 F}$ and $\mathscr{R}_{0 M F}$ use the extreme assumption in the early dengue epidemic that the dynamics of an infected human are not yet intervened by the presence of an infected mosquito's influence, and vice versa.

The second construction of $\mathscr{R}_{0}$ estimation is done in order to revise the first construction. We assume that the exponential growth of the infective host, at the early state of infection, is slightly affected by the growth of the infective vector, and vice versa. The main problem here is to find the best fit interval (here we use the terminology take-off period) where exponential growth occurs. Statistically, this situation can be related to the linear phase fit in a short time period.

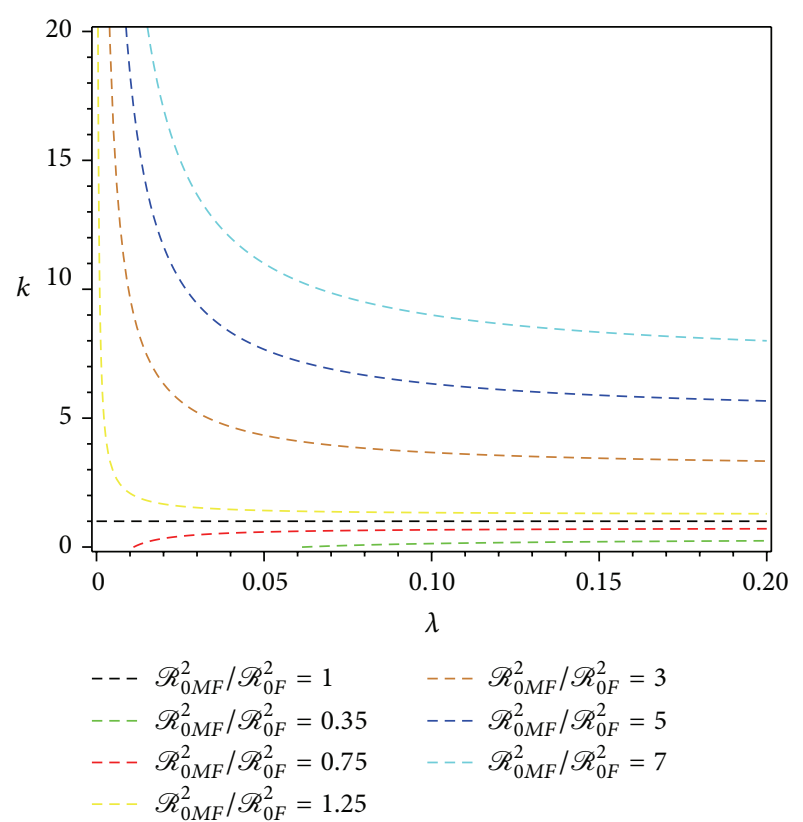

FIgURE 2: Level set for $\mathscr{R}_{0 M F}^{2} / \mathscr{R}_{0 F}^{2}$ in (15) for data set $\left\{\mu_{v}=1 / 30, \lambda \in\right.$ $[0,0.20], k \in[0,20]\}$.

We define the initial growth in a period of time as the initial value problem; that is,

$$
\begin{aligned}
I_{h}(t)= & I_{v 0}\left(a e^{\eta t}+b e^{-\eta t}\right) \\
& +I_{h 0}\left(c e^{\eta t}+d e^{-\eta t}\right), \\
I_{v}(t)= & I_{h 0}\left(p e^{\eta t}+q e^{-\eta t}\right) \\
& +I_{v 0}\left(r e^{\eta t}+s e^{-\eta t}\right) \\
\left(I_{h}(0), I_{v}(0)\right)= & \left(I_{h 0}, I_{v 0}\right) \\
\left(I_{h}^{\prime}(0), I_{v}^{\prime}(0)\right)= & \left(\eta I_{v 0}, \eta I_{h 0}\right) .
\end{aligned}
$$

We obtain

$$
\begin{aligned}
\{a & =\frac{1}{2}, b=\frac{1}{2}, c=\frac{1}{2}, d=\frac{1}{2}, p=\frac{1}{2}, q=\frac{1}{2}, r=\frac{1}{2}, s \\
& \left.=\frac{1}{2}\right\}
\end{aligned}
$$

such that the solution of (16) is obtained; namely,

$$
\begin{aligned}
& I_{h}(t)=I_{h 0} \cosh (\eta t)+I_{v 0} \sinh (\eta t), \\
& I_{v}(t)=I_{v 0} \cosh (\eta t)+I_{h 0} \sinh (\eta t) .
\end{aligned}
$$

The main idea from (18) is to extract the relevant parameter(s) during the early state of infection, in which linear growth rate of infection is assumed to take place. Next we substitute (18) with the expression for the derivative of $I_{h}$ and $I_{v}$ in (1) by simple algebraic manipulation; we then obtain 
a homogeneous system of equations in $\cosh (\eta t)$ and $\sinh (\eta t)$, namely,

$$
A\left[\begin{array}{c}
\cosh (\eta t) \\
\sinh (\eta t)
\end{array}\right]=\left[\begin{array}{l}
0 \\
0
\end{array}\right]
$$

where coefficient matrix is

$$
\begin{aligned}
& A \\
& =\left[\begin{array}{cc}
I_{h 0} \eta-b p_{h} I_{h 0}+\left(\gamma+\mu_{h}\right) I_{v 0} & I_{v 0} \eta-b p_{h} I_{v 0}+\left(\gamma+\mu_{h}\right) I_{h 0} \\
I_{v 0} \eta-b p_{v} \rho I_{v 0}+\mu_{v} I_{h 0} & I_{h 0} \eta-b p_{v} \rho I_{h 0}+\mu_{v} I_{v 0}
\end{array}\right] .
\end{aligned}
$$

A nontrivial solution from (19) exists if $|A|=0$, which gives

$$
\begin{aligned}
& \eta^{2} N_{h}-\left(b p_{v} N_{v}+N_{h} b p_{h}\right) \eta-\mu_{v}\left(\gamma+\mu_{h}\right) N_{h} \\
& +b^{2} p_{v} N_{v} p_{h}
\end{aligned}
$$

as the coefficient of $I_{h 0}^{2}$ and $I_{v 0}^{2}$ must be equal to zero. Equivalently, from (21), we obtain

$$
\begin{aligned}
\mathscr{R}_{0 A}^{2} & =-\frac{\eta^{2}}{\mu_{v}\left(\gamma+\mu_{h}\right)}+\frac{b\left(p_{h}+p_{v}\left(N_{v} / N_{h}\right)\right)}{\mu_{v}\left(\gamma+\mu_{h}\right)} \eta+1 \\
& =\frac{b^{2} p_{h} p_{v}}{\mu_{v}\left(\mu_{h}+\gamma\right)} \frac{N_{v}}{N_{h}}=\mathscr{R}_{0}^{2} .
\end{aligned}
$$

Therefore we have

$$
\mathscr{R}_{0 A}=\sqrt{-\frac{\eta^{2}}{\mu_{v}\left(\gamma+\mu_{h}\right)}+\frac{b\left(p_{h}+p_{v} \rho\right)}{\mu_{v}\left(\gamma+\mu_{h}\right)} \eta+1},
$$

where $\rho=N_{v} / N_{h}$ is the known mosquitoes per person index. We obtain (23), as a new construction for estimation $\mathscr{R}_{0}$, as a function of $\eta$. The construction of $\mathscr{R}_{0}$ in (23) is related to a short time interval where linear growth may still take place. Here, we will estimate the value of $\eta$ during the early state of infection, in which a linear growth of infection is assumed to take place.

Note that $\mathscr{R}_{0 A}$ increases with respect to $\eta, 0 \leq \eta \leq$ $b\left(p_{h}+p_{v} \rho\right) / 2$. Based on the definition that $\eta$ is probability, per unit of time, a susceptible becomes infected at the beginning of a dengue epidemic (see [7]), with assumption that $b\left(p_{h}+\right.$ $\left.p_{v} \rho\right) / 2<1$. This assumption means that the maximum takeoff rate of dengue virus infection is defending on the index mosquito per human. This condition implies

$$
\frac{2 \eta-b p_{h}}{b p_{v}} \leq \rho \leq \frac{2-b p_{h}}{b p_{v}} .
$$

$\mathscr{R}_{0 A} / \mathscr{R}_{0 F}$ ratio in the early phase of dengue epidemics could be significantly larger or smaller than one depending on the value of $\rho$, in (24), and the value of $\lambda$. In Section 4, we determine $\eta$ as a function of $\lambda$ at the beginning of a dengue epidemic. We will further analyze the level set of the ratio $\mathscr{R}_{0 A} / \mathscr{R}_{0 F}$. Considering that the value of $\mathscr{R}_{0}$ estimation depends on the value of take-off rate $\lambda$, we discuss a method to formulate the take-off rate in the following section.

\section{Formulation of the Take-Off Rate}

Here we derive two scenarios for the derivation of the take-off rate (t.o.r.) at the beginning of every take-off period (t.o.p.) of dengue infection. Both scenarios are based on the same assumptions, but with a slightly different implementation for real-life dengue epidemics. Therefore, the main problem here is to find the best fit interval (here we use the terminology "take-off period") where exponential growth occurs.

The first scenario is done under the assumption that, during early infection, recovery and natural death do not yet occur. Note that the original data taken from the hospital is in the form of daily new cases. Let $I(t)$ be the number of new cases of infection; we then have $I(t)=d I_{h} / d t$ (see in [14]). Therefore, from (7), it is

$$
I(t)=\lambda I_{h 0} e^{\lambda t}, \quad \text { with } I_{0}=I(0)=\lambda I_{h 0}
$$

and, from (12), it is

$$
\begin{aligned}
& I(t)=\eta\left(I_{v 0} \cosh (\eta t)+I_{h 0} \sinh (\eta t)\right), \\
& \text { with } I_{0}=I(0)=\eta I_{v 0} .
\end{aligned}
$$

By using the Taylor expansion around $\lambda=0$ for (25) and around $\eta=0$ for (26), we obtain

$$
\begin{aligned}
& I(t) \approx \lambda I_{0} t+I_{0}, \\
& I(t) \approx \frac{\eta^{2}}{\lambda} I_{0} t+I_{0},
\end{aligned}
$$

respectively. Consequently, we have a relation that $\lambda=\eta$. Thus $\eta$ at $\mathscr{R}_{0 A}$ in (23) can be replaced by $\lambda$. By comparing (11) and (23), we have

$$
\frac{\mathscr{R}_{0 A}^{2}}{\mathscr{R}_{0 F}^{2}}=1+\frac{\lambda}{\lambda+\mu_{v}}\left(\frac{b\left(p_{h}+p_{v} \rho\right)-\lambda}{\lambda+\mu_{h}+\gamma}-1\right) .
$$

Also $\eta$ in (24) can be replaced by $\lambda$ such that we obtain

$$
\frac{2 \lambda-b p_{h}}{b p_{v}} \leq \rho \leq \frac{2-b p_{h}}{b p_{v}} .
$$

The ratio $\mathscr{R}_{0 A}^{2} / \mathscr{R}_{0 F}^{2} \geq 1$, if and only if $\rho \geq\left(2 \lambda+\mu_{h}+\gamma-\right.$ $\left.b p_{h}\right) / b p_{v}$, and $\mathscr{R}_{0 A}^{2} / \mathscr{R}_{0 F}^{2}<1$, if and only if $\rho<\left(2 \lambda+\mu_{h}+\gamma-\right.$ $\left.b p_{h}\right) / b p_{v}$. Because of $\left(2 \lambda-b p_{h}\right) / b p_{v}<\left(2 \lambda+\mu_{h}+\gamma-b p_{h}\right) / b p_{v}$, we have $\left(2 \lambda+\mu_{h}+\gamma-b p_{h}\right) / b p_{v}$, which belongs to the range of $\rho$ for small $\lambda$.

The second scenario is conducted under the same assumption as the first scenario. But, here, we regress between the number of new cases in a day $I(t)=d I_{h} / d t$ and cumulative number of new cases $K(t)$, where we have the initial value $K(0)=K_{0}=I_{0}=I(0)=\lambda I_{h 0}$ from (7) and $K(0)=K_{0}=I_{0}=$ $I(0)=\eta I_{v 0}$ from (18). Therefore, by using integral equation $K(t)-K(0)=\int_{0}^{t} I(\tau) d \tau$ with $I(\tau)$ from the first equation in (7) and $I(\tau)$ from the first equation in (18), we obtain

$$
\begin{aligned}
& I(t)=\lambda K(t)+(1-\lambda) K_{0}, \\
& I(t)=\eta^{2} K(t)+\left(1-\eta^{2}\right) K_{0},
\end{aligned}
$$




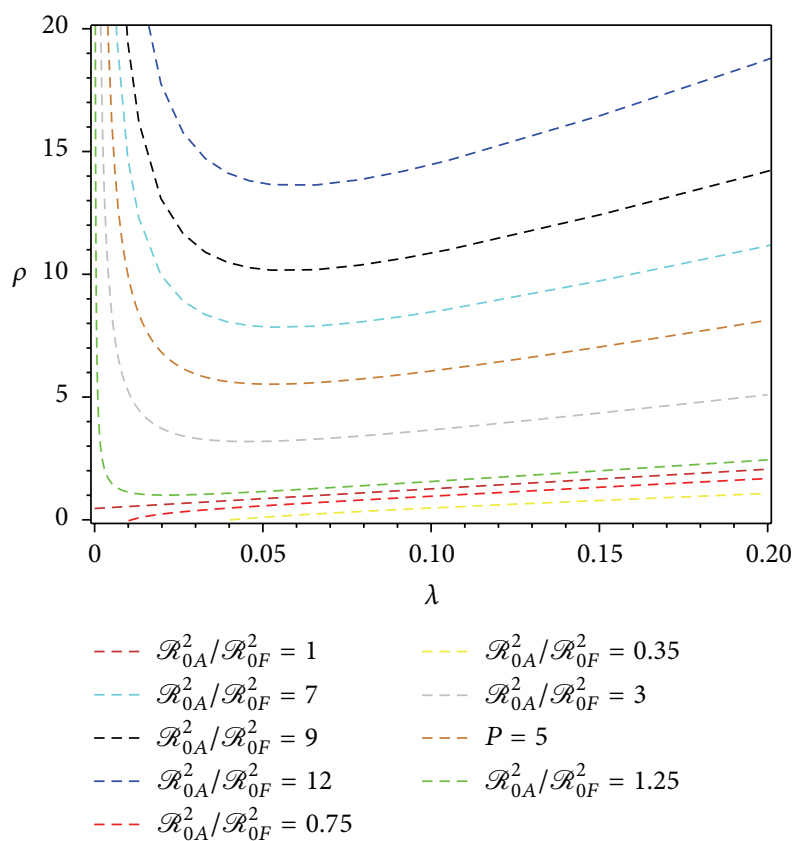

Figure 3: Level set $\mathscr{R}_{0 A}^{2} / \mathscr{R}_{0 F}^{2}$ in (28) for data set $\left\{b=1, p_{h}=0.01\right.$, $p_{v}=0.25, \gamma=1 / 8, \mu_{h}=1 /(3 \times 70), \mu_{v}=1 / 30, \lambda \in[0,0.20], \rho \in$ $[0,20]\}$.

respectively. It is clear that $\eta^{2}=\lambda$ so that $\eta$ in $\mathscr{R}_{0 A}$ at (23) can be replaced by $\sqrt{\lambda}$. If (23) is divided by (11), we obtain

$$
\frac{\mathscr{R}_{0 A}^{2}}{\mathscr{R}_{0 F}^{2}}=1+\frac{\sqrt{\lambda}}{\lambda+\mu_{v}}\left(\frac{b\left(p_{h}+p_{v} \rho\right)-\sqrt{\lambda}}{\lambda+\mu_{h}+\gamma}-\sqrt{\lambda}\right) .
$$

Also $\eta$ in (24) can be replaced by $\sqrt{\lambda}$ such that we obtain

$$
\frac{2 \sqrt{\lambda}-b p_{h}}{b p_{v}} \leq \rho \leq \frac{2-b p_{h}}{b p_{v}} .
$$

We note that the ratio $\mathscr{R}_{0 A}^{2} / \mathscr{R}_{0 F}^{2} \geq 1$ if and only if $\rho \geq\left(\lambda^{3 / 2}+\right.$ $\left.\lambda^{1 / 2}\left(\mu_{v}+\mu_{h}+\gamma+1\right)-b p_{h}\right) / b p_{v}=\rho_{0}$ and $\mathscr{R}_{0 A}^{2} / \mathscr{R}_{0 F}^{2}<1$ if and only if $\rho<\rho_{0}$. Note also that $\rho_{0}<\left(2-b p_{h}\right) / b p_{v}$ for small $\lambda$.

Figures 3 and 4 show the level sets for some values of the ratio $\mathscr{R}_{0 A}^{2} / \mathscr{R}_{0 F}^{2}$, for both scenarios of construction of $\lambda$. It shows that for small $\lambda, \mathscr{R}_{0 A}^{2} / \mathscr{R}_{0 F}^{2}$ increases faster, with respect to $\rho$, in the second scenario (Figure 4) than in the first scenario (Figure 3). Summary of the construction of $\mathscr{R}_{0}$ estimation for the first and the second scenario of $\lambda$ is given in Table 1.

The magnitude of $\mathscr{R}_{0 A}$ in Figure 5 indicates that the second scenario is rising faster than $\mathscr{R}_{0 A}$ of the first scenario at the beginning of the growing epidemic of dengue fever, although the rate of infection is very small. This means that the presence of mosquitoes in early dengue infection directly affects the human dengue infection. Furthermore, Figure 5 shows that the second scenario of construction of $\mathscr{R}_{0}$, which is summarized in Table 1, is increasing faster than the first scenario. Therefore, we obtain that the second scenario is a more realistic construction of $\mathscr{R}_{0}$ than the first one at the beginning of the short period of dengue virus infection.

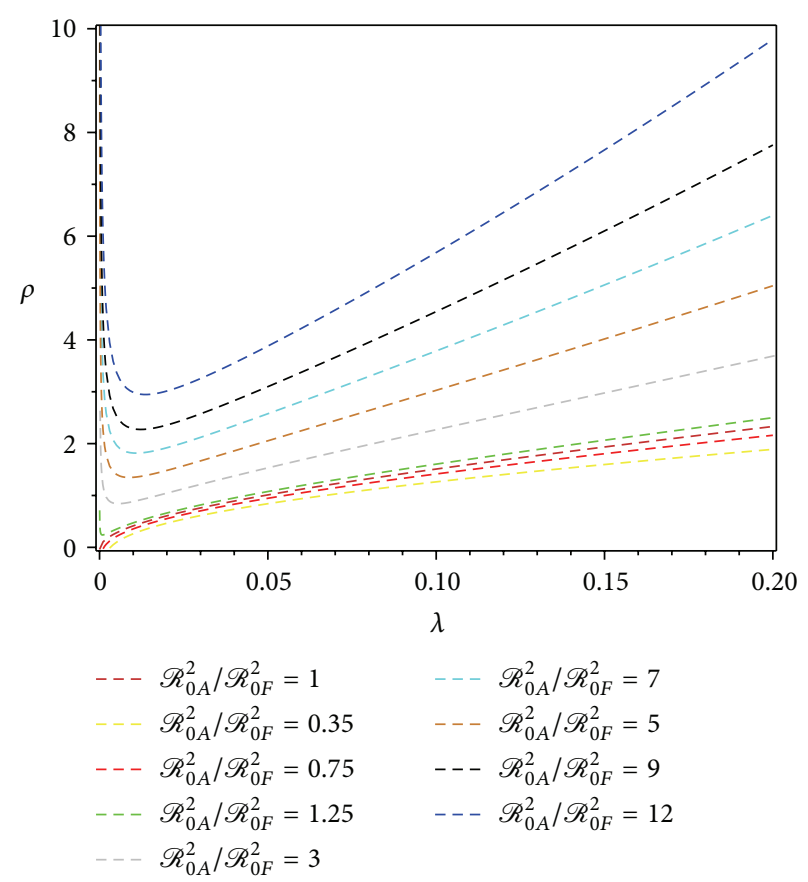

FIGURE 4: Level set $\mathscr{R}_{0 A}^{2} / \mathscr{R}_{0 F}^{2}$ in (31) for data set $\left\{b=1, p_{h}=0.01\right.$, $p_{v}=0.25, \gamma=1 / 8, \mu_{h}=1 /(3 \times 70), \mu_{v}=1 / 30, \lambda \in[0,0.20], \rho \in$ $[0,10]\}$.

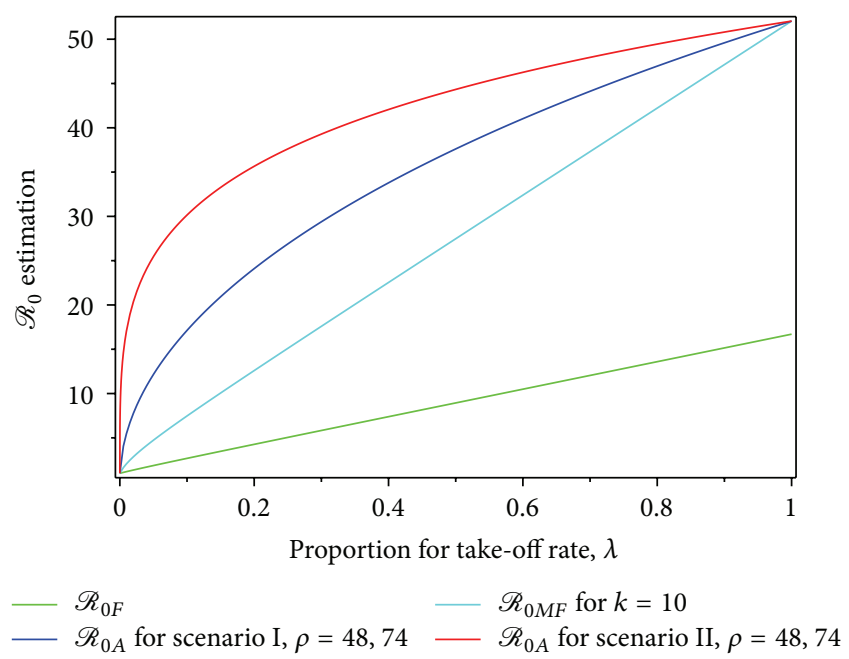

FIGURE 5: The comparison of $\mathscr{R}_{0}$ estimation.

In the next section, we apply least square method to determine estimation of $\lambda, I_{0}$, and $K_{0}$ from dengue incidence data based on (27) and (30). We calculated $\lambda, I_{0}$, and $K_{0}$ by using least square method which differs from the estimation that has been conducted in [14]. They assume that the number of cases of dengue at $t=0$ is equal to one, but, here, we estimate from the dengue incidence data. In the next section, we examine the dependence of the basic reproductive ratio on the take-off rate, at the beginning of the take-off period of dengue infection, for daily cases of dengue from SB Hospital in Bandung Indonesia. 
TABLE 1: Summary of the construction of $\mathscr{R}_{0}$ and $\lambda$ estimation for the first scenario.

\begin{tabular}{|c|c|}
\hline The first scenario for $\lambda$ & $\mathscr{R}_{0}$ estimation \\
\hline Based on [12] assumption & $\mathscr{R}_{0 F}^{2}=\frac{\lambda^{2}}{\mu_{v}\left(\mu_{h}+\gamma\right)}+\frac{\left(\mu_{v}+\mu_{h}+\gamma\right) \lambda}{\mu_{v}\left(\mu_{h}+\gamma\right)}+1$ \\
\hline The first construction & $\mathscr{R}_{0 M F}^{2}=\frac{k \lambda^{2}}{\mu_{v}\left(\mu_{h}+\gamma\right)}+\frac{\left(\mu_{v}+k\left(\mu_{h}+\gamma\right)\right) \lambda}{\mu_{v}\left(\mu_{h}+\gamma\right)}+1$ \\
\hline The second construction & $\mathscr{R}_{0 A}^{2}=-\frac{\lambda^{2}}{\mu_{v}\left(\gamma+\mu_{h}\right)}+\frac{b\left(p_{h}+p_{v} \rho\right)}{\mu_{v}\left(\gamma+\mu_{h}\right)} \lambda+1$ \\
\hline The t.o.r. $\lambda$ equation & $I(t)=\lambda I_{0} t+I_{0}$ \\
\hline The second scenario for $\lambda$ & $\mathscr{R}_{0}$ estimation \\
\hline Based on [12] assumption & $\mathscr{R}_{0 F}^{2}=\frac{\lambda^{2}}{\mu_{v}\left(\mu_{h}+\gamma\right)}+\frac{\left(\mu_{v}+\mu_{h}+\gamma\right) \lambda}{\mu_{v}\left(\mu_{h}+\gamma\right)}+1$ \\
\hline The first construction & $\mathscr{R}_{0 M F}^{2}=\frac{\kappa \Lambda}{\mu_{v}\left(\mu_{h}+\gamma\right)}+\frac{\left(\mu_{v}+\kappa\left(\mu_{h}+\gamma\right)\right) \Lambda}{\mu_{v}\left(\mu_{h}+\gamma\right)}+1$ \\
\hline The second construction & $\mathscr{R}_{0 A}^{2}=-\frac{\lambda}{\mu_{v}\left(\gamma+\mu_{h}\right)}+\frac{b\left(p_{h}+p_{v} \rho\right)}{\mu_{v}\left(\gamma+\mu_{h}\right)} \sqrt{\lambda}+1$ \\
\hline The t.o.r. $\lambda$ equation & $I(t)=\lambda K(t)+(1-\lambda) K_{0}$ \\
\hline
\end{tabular}

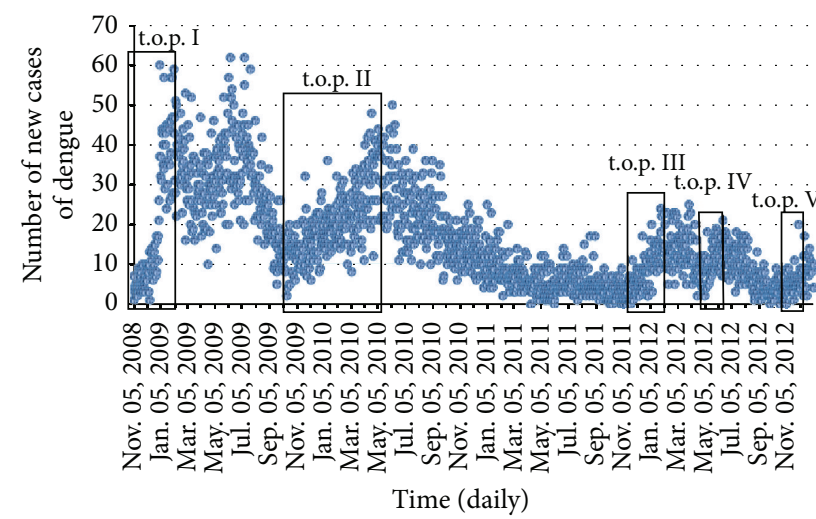

FIGURE 6: A time series of the number of daily new cases of dengue infection in the SB Hospital is from Nov. 05, 2008, to Dec. 2012. The cut off period of new cases of dengue is inside the solid box. Every t.o.p. contains i.t.o.p. of dengue infection.

\section{Application of $\mathscr{R}_{0}$ Estimation to the Real-Life Dengue Epidemic}

This section presents the application of the method to the data of dengue incidence from SB hospital, the estimation value of the t.o.r. $\lambda$, the value basic reproductive ratio, and their implication. Calculation of $\lambda$ is based on the assumption that at the beginning of the infection natural death and recovery have not yet occurred. This assumption can be taken before the eighth day of incidence. Data is divided into five takeoff periods (t.o.p.) of infections, which is represented inside the solid box in Figure 6. Each t.o.p. contains an initial takeoff period (i.t.o.p.) of dengue infection. Here, we define the i.t.o.p. as being within the range of the fourth and the seventh days of the dengue incidence. The correspondents of the bioepidemiological parameter from human and mosquito are given in Table 2.

Figure 6 shows the five possibilities for the t.o.p. The t.o.p. is defined by the minimum to maximum values of $r$-square
TABLE 2: Bioepidemiological data of human and mosquito.

\begin{tabular}{lcc}
\hline Symbol & Definition & Value \\
\hline$b$ & Biting rate $\left(\right.$ day $\left.^{-1}\right)$ & 1 \\
$p_{h}$ & Effective contact rate to human & 0.01 \\
$p_{v}$ & Effective contact rate to mosquito & 0.25 \\
$\mu_{h}$ & Human mortality rate $\left(\right.$ day $\left.^{-1}\right)$ & $\frac{1}{365 \times 70}$ \\
$\mu_{v}$ & Mosquito mortality rate $\left(\right.$ day $\left.^{-1}\right)$ & $\frac{1}{30}$ \\
$\gamma$ & Recovering rate of human $\left(\right.$ day $\left.^{-1}\right)$ & $\frac{1}{8}$ \\
$\rho$ & Mosquito per human index & $0-7.96$ \\
$k$ & Proportion of mosquito per human index & $0-2$ \\
\hline
\end{tabular}

$\left(r^{2}\right)$. The number of days for every i.t.o.p. of incidence data depends on the value of $r^{2}$ criteria (approximately at the 47 th day after contact with the infected mosquito). Meanwhile, the i.t.o.p. is defined by the minimum to maximum values of $r^{2}$ before the eighth day. Next we determine the rate of the i.t.o.p. of dengue infection (t.o.r.) $\lambda$ for every t.o.p. The value of $\lambda$ is calculated at intervals of four to seven days for the dengue of dengue infection by using two different scenarios in respect to the incidence data and the value of parameter in Table 2.

In the first scenario we calculate the values of $\lambda$ and $I_{0}$ by using least square method for (27), and, in the second scenario, we calculate the values of $\lambda$ and $K_{0}$ by using least square method for (30), for every i.t.o.p. Those values of $\lambda, I_{0}$, and $K_{0}$ are summarized in Table 3.

Using take-off rate values of infection $\lambda$ in Table 3 , we calculate the value of $\rho, \mathscr{R}_{0 F}, \mathscr{R}_{0 M F}$, and $\mathscr{R}_{0 A}$, which are given in Tables $4-7$ as well as the intervals of $\widehat{\rho}, \widehat{\mathscr{R}}_{0 M F}, \widehat{\mathscr{R}}_{0}$, 
TABLE 3: The value of $\lambda$ and $I_{0}$ from the first scenario and the value of $\lambda$ and $K_{0}$ for the second scenario, respectively.

\begin{tabular}{lccc}
\hline Take-off period & Initial take-off period & $\begin{array}{c}\text { The values of } \lambda \text { and } I_{0} \text { for } \\
\text { the first scenario }\end{array}$ & $\begin{array}{c}\text { The values of } \lambda \text { and } K_{0} \text { for } \\
\text { the second scenario }\end{array}$ \\
\hline I: 103 days & I: 5 days & $\lambda=0.093, I_{0}=5.4$ & $\lambda=0.091, K_{0}=5.22$ \\
II: 187 days & II: 6 days & $\lambda=0.009, I_{0}=11.53$ & $\lambda=0.019, K_{0}=11.17$ \\
III: 13 days & III: 6 days & $\lambda=0.190, I_{0}=3.62$ & $\lambda=0.121, K_{0}=3.78$ \\
IV: 17 days & IV: 5 days & $\lambda=0.038, I_{0}=16$ & $\lambda=0.048, K_{0}=15.54$ \\
V: 85 days & V: 7 days & $\lambda=0.171, I_{0}=2.93$ & $\lambda=0.105, K_{0}=3.1$ \\
\hline
\end{tabular}

TABLE 4: The values of $\mathscr{R}_{0 F}$ for a given value of $\lambda$ at the i.t.o.p. and the corresponding values of $\mathscr{R}_{0 M F}$ for different values of $k$ for the first scenario.

\begin{tabular}{lcccccc}
\hline The $n$ days at the i.t.o.p. & $\lambda$ & $\mathscr{R}_{0 F}$ & $\begin{array}{c}\mathscr{R}_{0 M F} \\
k=0\end{array}$ & $\begin{array}{c}\mathscr{R}_{0 M F} \\
k=0.75\end{array}$ & $\begin{array}{c}\mathscr{R}_{0 M F} \\
k=1\end{array}$ & $\begin{array}{c}\mathscr{R}_{0 M F} \\
k=1.25\end{array}$ \\
\hline II: 7 days & 0.009 & {$[1.17]$} & 1.04 & 1.14 & {$[1.17]$} & 1.20 \\
IV: 5 days & 0.038 & {$[1.66]$} & 1.14 & 1.55 & {$[1.66]$} & 1.77 \\
I: 5 days & 0.093 & {$[2.56]$} & 1.32 & 2.32 & {$[2.56]$} & 2.79 \\
V: 7 days & 0.171 & {$[3.80]$} & 1.54 & 3.38 & {$[3.80]$} & 4.18 \\
III: 6 days & 0.190 & {$[3.89]$} & 1.59 & 3.64 & {$[3.89]$} & 4.52 \\
\hline
\end{tabular}

TABLE 5: The values of $\mathscr{R}_{0 F}$ for a given value of $\lambda$ at the i.t.o.p. and the corresponding values of $\mathscr{R}_{0 M F}$ for different values of $k$ for the second scenario.

\begin{tabular}{lcccccc}
\hline The $n$ days at the i.t.o.p. & $\lambda$ & $\mathscr{R}_{0 F}$ & $\begin{array}{c}\mathscr{R}_{0 M F} \\
k=0\end{array}$ & $\begin{array}{c}\mathscr{R}_{0 M F} \\
k=0.75\end{array}$ & $\begin{array}{c}\mathscr{R}_{0 M F} \\
k=1\end{array}$ & $\begin{array}{c}\mathscr{R}_{0 M F} \\
k=1.25\end{array}$ \\
\hline II: 7 days & 0.019 & {$[1.35]$} & 1.07 & 1.29 & {$[1.35]$} & 1.41 \\
IV: 5 days & 0.048 & {$[1.83]$} & 1.17 & 1.69 & {$[1.83]$} & 1.96 \\
I: 5 days & 0.091 & {$[2.54]$} & 1.32 & 2.30 & {$[2.54]$} & 2.77 \\
V: 7 days & 0.105 & {$[2.76]$} & 1.36 & 2.49 & {$[2.76]$} & 3.01 \\
III: 6 days & 0.121 & {$[3.02]$} & 1.40 & 2.71 & {$[3.02]$} & 3.30 \\
\hline
\end{tabular}

TABLE 6: The values of $\mathscr{R}_{0 F}$ for every value of $\lambda$ at each initial t.o.p. and the values of $\mathscr{R}_{0 A}$ for every change in the value of $\lambda$ and $\rho$ for the first scenario for calculation of $\lambda$.

\begin{tabular}{lccccccc}
\hline The $n$ days at the i.t.o.p. & $\lambda$ & $\mathscr{R}_{0 F}$ & $\begin{array}{c}\mathscr{R}_{0 A} \\
\rho=0.67\end{array}$ & $\begin{array}{c}\mathscr{R}_{0 A} \\
\rho=0.89\end{array}$ & $\begin{array}{c}\mathscr{R}_{0 A} \\
\rho=1.33\end{array}$ & $\begin{array}{c}\mathscr{R}_{0 A} \\
\rho=1.96\end{array}$ & $\rho=2.11$ \\
\hline II: 7 days & 0.009 & {$[1.17]$} & {$[1.17]$} & 1.22 & 1.32 & 1.45 \\
IV: 5 days & 0.038 & {$[1.66]$} & 1.50 & {$[1.66]$} & 1.94 & 2.27 \\
I: 5 days & 0.093 & {$[2.56]$} & 1.70 & 2.03 & {$[2.56]$} & 3.17 & 2.34 \\
V: 7 days & 0.171 & {$[3.80]$} & 1.12 & 1.89 & 2.84 & {$[3.80]$} & 4.00 \\
III: 6 days & 0.190 & {$[4.10]$} & 0.67 & 1.73 & 2.83 & 3.89 & {$[4.10]$} \\
\hline
\end{tabular}

TABLE 7: The values of $\mathscr{R}_{0 F}$ for every value of $\lambda$ at each initial t.o.p. and the values of $\mathscr{R}_{0 A}$ for every change in the value of $\lambda$ and $\rho$ for the second scenario for calculating of $\lambda$.

\begin{tabular}{|c|c|c|c|c|c|c|c|}
\hline The $n$ days at the i.t.o.p. & $\lambda$ & $\mathscr{R}_{0 F}$ & $\begin{array}{c}\mathscr{R}_{0 A} \\
\rho=0.61\end{array}$ & $\begin{array}{c}\mathscr{R}_{0 A} \\
\rho=1.01\end{array}$ & $\begin{array}{c}\mathscr{R}_{0 A} \\
\rho=1.47\end{array}$ & $\begin{array}{c}\mathscr{R}_{0 A} \\
\rho=1.60\end{array}$ & $\begin{array}{c}\mathscr{R}_{0 A} \\
\rho=1.74\end{array}$ \\
\hline II: 7 days & 0.019 & {$[1.35]$} & {$[1.35]$} & 2.27 & 2.99 & 3.16 & 3.34 \\
\hline IV: 5 days & 0.048 & {$[1.83]$} & - & {$[1.83]$} & 3.06 & 3.32 & 3.59 \\
\hline I: 5 days & 0.091 & {$[2.54]$} & - & - & {$[2.54]$} & 2.96 & 3.37 \\
\hline V: 7 days & 0.105 & {$[2.76]$} & - & - & 2.27 & {$[2.76]$} & 3.23 \\
\hline III: 6 days & 0.121 & {$[3.02]$} & - & - & 1.87 & 2.48 & {$[3.02]$} \\
\hline
\end{tabular}


TABLE 8: Summary of the $\widehat{\mathscr{R}}_{0}$ and $\widehat{\mathscr{R}}_{0 M F}$ for the first scenario of the value of $\lambda$ and $\rho$ in (28).

\begin{tabular}{|c|c|c|c|c|c|}
\hline The $n$ days at the i.t.o.p. & $\lambda$ & $\begin{array}{c}\hat{\rho} \\
\text { from }(28)\end{array}$ & $\begin{array}{c}\widehat{\mathscr{R}}_{0 M F} \\
\text { for } k \in(0,2]\end{array}$ & $\widehat{\mathscr{R}}_{0}$ & Important notes \\
\hline II: 7 days & 0.009 & $(0.034,7.96)$ & $(1.04,1.29]$ & $(0.02,4.77)$ & $\mathscr{R}_{0 F} \in \widehat{\mathscr{R}}_{0 M F} \subset \widehat{\mathscr{R}}_{0}$ \\
\hline IV: 5 days & 0.038 & $(0.26,7.96)$ & $(1.14,2.06]$ & $(0.16,4.77)$ & $\mathscr{R}_{0 F} \in \widehat{\mathscr{R}}_{0 M F} \subset \widehat{\mathscr{R}}_{0}$ \\
\hline I: 5 days & 0.093 & $(0.701,7.96)$ & $(1.32,3.38]$ & $(0.42,4.77)$ & $\mathscr{R}_{0 F} \in \widehat{\mathscr{R}}_{0 M F} \subset \widehat{\mathscr{R}}_{0}$ \\
\hline V: 7 days & 0.171 & $(1.33,7.96)$ & $(1.54,5.16]$ & $(0.80,4.77)$ & $\mathscr{R}_{0 F} \in \widehat{\mathscr{R}}_{0} \cap \widehat{\mathscr{R}}_{0 M F}$ \\
\hline III: 6 days & 0.190 & $(1.48,7.96)$ & $(1.59,5.58]$ & $(0.89,4.77)$ & $\mathscr{R}_{0 F} \in \widehat{\mathscr{R}}_{0} \cap \widehat{\mathscr{R}}_{0 M F}$ \\
\hline
\end{tabular}

Note. The value of $\mathscr{R}_{0 F}$ from Table 6.

TABLE 9: Summary of the $\widehat{\mathscr{R}}_{0}$ and $\widehat{\mathscr{R}}_{0 M F}$ for the second scenario of the value of $\lambda$ and $\rho$ in (31).

\begin{tabular}{|c|c|c|c|c|c|}
\hline The $n$ days at the i.t.o.p. & $\lambda$ & $\begin{array}{c}\widehat{\rho} \\
\text { from }(31)\end{array}$ & $\widehat{\mathscr{R}}_{0}$ & $\begin{array}{c}\widehat{\mathscr{R}}_{0 M F} \\
\text { for } k \in(0,2]\end{array}$ & Important notes \\
\hline II : 7 days & 0.019 & $(1.07,7.96)$ & $(0.64,4.77)$ & $(1.07,1.58)$ & $\mathscr{R}_{0 F} \in \widehat{\mathscr{R}}_{0 M F} \subset \widehat{\mathscr{R}}_{0}$ \\
\hline IV: 5 days & 0.048 & $(1.71,7.96)$ & $(1.02,4.77)$ & $(1.17,2.30)$ & $\mathscr{R}_{0 F} \in \widehat{\mathscr{R}}_{0 M F} \subset \widehat{\mathscr{R}}_{0}$ \\
\hline I: 5 days & 0.091 & $(2.37,7.96)$ & $(1.43,4.77)$ & $(1.32,3.35)$ & $\mathscr{R}_{0 F} \in \widehat{\mathscr{R}}_{0} \cap \widehat{\mathscr{R}}_{0 M F}$ \\
\hline V: 7 days & 0.105 & $(2.55,7.96)$ & $(1.53,4.77)$ & $(1.36,3.66)$ & $\mathscr{R}_{0 F} \in \widehat{\mathscr{R}}_{0} \cap \widehat{\mathscr{R}}_{0 M F}$ \\
\hline III: 6 days & 0.121 & $(2.74,7.96)$ & $(1.65,4.77)$ & $(1.40,4.03)$ & $\mathscr{R}_{0 F} \in \widehat{\mathscr{R}}_{0} \cap \widehat{\mathscr{R}}_{0 M F}$ \\
\hline
\end{tabular}

Note. The value of $\mathscr{R}_{0 F}$ from Table 7.

TABLE 10: Summary of the $\mathscr{R}_{0}$ and $\mathscr{R}_{0 A}$ for the first scenario of the value of $\lambda$ and $\rho$ in (28).

\begin{tabular}{|c|c|c|c|c|c|}
\hline The $n$ days at the i.t.o.p. & $\lambda$ & $\begin{array}{c}\widehat{\rho} \\
\text { from }(28)\end{array}$ & $\widehat{\mathscr{R}}_{0}$ & $\widehat{\mathscr{R}}_{0 A}$ & Important notes \\
\hline II: 7 days & 0.009 & $(0.034,7.96)$ & $(0.02,4.77)$ & $(1.01,2.33)$ & $\mathscr{R}_{0 F} \in \widehat{\mathscr{R}}_{0 A} \subset \widehat{\mathscr{R}}_{0}$ \\
\hline IV: 5 days & 0.038 & $(0.26,7.96)$ & $(0.16,4.77)$ & $(1.16,4.32)$ & $\mathscr{R}_{0 F} \in \widehat{\mathscr{R}}_{0 A} \subset \widehat{\mathscr{R}}_{0}$ \\
\hline I: 5 days & 0.093 & $(0.701,7.96)$ & $(0.42,4.77)$ & $(1.75,6.59)$ & $\mathscr{R}_{0 F} \in \widehat{\mathscr{R}}_{0} \cap \widehat{\mathscr{R}}_{0 A}$ \\
\hline V: 7 days & 0.171 & $(1.33,7.96)$ & $(0.80,4.77)$ & $(2.83,8.71)$ & $\mathscr{R}_{0 F} \in \widehat{\mathscr{R}}_{0} \cap \widehat{\mathscr{R}}_{0 A}$ \\
\hline III: 6 days & 0.190 & $(1.48,7.96)$ & $(0.89,4.77)$ & $(3.10,9.13)$ & $\mathscr{R}_{0 F} \in \widehat{\mathscr{R}}_{0} \cap \widehat{\mathscr{R}}_{0 A}$ \\
\hline
\end{tabular}

Note. The value of $\mathscr{R}_{0 F}$ from Table 6.

TABLE 11: Summary of the $\widehat{\mathscr{R}}_{0}$ and $\widehat{\mathscr{R}}_{0 A}$ for the second scenario of the value of $\lambda$ and $\rho$ in (31).

\begin{tabular}{|c|c|c|c|c|c|}
\hline The $n$ days at the i.t.o.p. & $\lambda$ & $\begin{array}{c}\hat{\rho} \\
\text { from }(31)\end{array}$ & $\widehat{\mathscr{R}}_{0}$ & $\widehat{\mathscr{R}}_{0 A}$ & Important notes \\
\hline II: 7 days & 0.019 & $(1.07,7.96)$ & $(0.64,4.77)$ & $(2.37,7.93)$ & $\mathscr{R}_{0 F} \in \widehat{\mathscr{R}}_{0} \backslash \widehat{\mathscr{R}}_{0 A}$ \\
\hline IV: 5 days & 0.048 & $(1.704,7.96)$ & $(1.02,4.77)$ & $(3.52,9.70)$ & $\mathscr{R}_{0 F} \in \widehat{\mathscr{R}}_{0} \backslash \widehat{\mathscr{R}}_{0 A}$ \\
\hline I: 5 days & 0.091 & $(2.37,7.96)$ & $(1.43,4.77)$ & $(4.79,11.14)$ & $\mathscr{R}_{0 F} \in \widehat{\mathscr{R}}_{0} \backslash \widehat{\mathscr{R}}_{0 A}$ \\
\hline V: 7 days & 0.105 & $(2.55,7.96)$ & $(1.53,4.77)$ & $(5.12,11.46)$ & $\mathscr{R}_{0 F} \in \widehat{\mathscr{R}}_{0} \backslash \widehat{\mathscr{R}}_{0 A}$ \\
\hline III: 6 days & 0.121 & $(2.74,7.96)$ & $(1.65,4.77)$ & $(5.48,11.78)$ & $\mathscr{R}_{0 F} \in \widehat{\mathscr{R}}_{0} \backslash \widehat{\mathscr{R}}_{0 A}$ \\
\hline
\end{tabular}

Note. The value of $\mathscr{R}_{0 F}$ from Table 7 .

and $\widehat{\mathscr{R}}_{0 A}$ and their important notes, which are given in Tables $8-13$.

The interpretation of the result in Tables 9-13, for instance, on $\mathscr{R}_{0 F}$ at t.o.p. III, is that each infective person infected 3.89 other persons via the mosquito vector at the start of the epidemic when the population was susceptible. It is similar meaning for other constructions of $\mathscr{R}_{0}$ estimation. The maximum basic reproductive ratio, $\mathscr{R}_{0 F}$ found was 4.10 , $\mathscr{R}_{0 M F}$ found was 5.58 , and $\mathscr{R}_{0 A}$ found was 9.13 , which should be compared with the maximum value which was obtained 103 in [12]. Furthermore, $\mathscr{R}_{0 F}$ is a special case for both $\mathscr{R}_{0 M F}$ and $\mathscr{R}_{0 A}$, in terms of magnitude. Furthermore, $\mathscr{R}_{0 M F}$ magnitude is a special case of $\mathscr{R}_{0 \mathrm{~A}}$ magnitude. $\mathscr{R}_{0 \mathrm{~A}}$ magnitude therefore generalized both $\mathscr{R}_{0 F}$ and $\mathscr{R}_{0 M F}$ magnitudes. Table 4 shows that the value of $\mathscr{R}_{0 F}$ increases with an increasing $\lambda$. Also, $\mathscr{R}_{0 M F}$ increases with increasing $\lambda$ and $k$. The special cases are $\mathscr{R}_{0 F}=\mathscr{R}_{0 M F}$ for $k=1, \mathscr{R}_{0 M F}<\mathscr{R}_{0 F}$ for $k<1$, and $\mathscr{R}_{0 F}<\mathscr{R}_{0 M F}$ for $k>1$. Thus, if we have the value of $k$ from the real-life situation, then the value of $\mathscr{R}_{0 M F}$ is more realistic for estimating $\mathscr{R}_{0}$ rather than $\mathscr{R}_{0 F}$.

From Tables 4 and 5, with respect to $\mathscr{R}_{0 F}$, we obtain that the average secondary case of dengue infection is at most 
TABLE 12: The value of $\mathscr{R}_{0 F}$ is related to $\widehat{\mathscr{R}}_{0 M F}, \widehat{\mathscr{R}}_{0}$, and $\widehat{\mathscr{R}}_{0 A}$ for the first scenario.

\begin{tabular}{|c|c|c|c|c|}
\hline The $n$ days at the i.t.o.p. & $\lambda$ & $\mathscr{R}_{0 F}$ & $\begin{array}{c}\widehat{\mathscr{R}}_{0 M F} \\
\text { for } k \in(0,2]\end{array}$ & Important notes \\
\hline II: 7 days & 0.009 & 1.17 & $(1.04,1.29]$ & $\mathscr{R}_{0 F} \in \widehat{\mathscr{R}}_{0 M F} \cap \widehat{\mathscr{R}}_{0} \cap \widehat{\mathscr{R}}_{0 A}$ \\
\hline IV: 5 days & 0.038 & 1.66 & $(1.14,2.06]$ & $\mathscr{R}_{0 F} \in \widehat{\mathscr{R}}_{0 M F} \cap \widetilde{\mathscr{R}}_{0} \cap \widehat{\mathscr{R}}_{0 A}$ \\
\hline I: 5 days & 0.093 & 2.56 & $(1.32,3.38]$ & $\mathscr{R}_{0 F} \in \widehat{\mathscr{R}}_{0 M F} \cap \widehat{\mathscr{R}}_{0} \cap \widehat{\mathscr{R}}_{0 A}$ \\
\hline V: 7 days & 0.171 & 3.80 & $(1.54,5.16]$ & $\mathscr{R}_{0 F} \in \widehat{\mathscr{R}}_{0 M F} \cap \widehat{\mathscr{R}}_{0} \cap \widehat{\mathscr{R}}_{0 A}$ \\
\hline III: 6 days & 0.190 & 3.89 & $(1.59,5.58]$ & $\mathscr{R}_{0 F} \in \widehat{\mathscr{R}}_{0 M F} \cap \widehat{\mathscr{R}}_{0} \cap \widehat{\mathscr{R}}_{0 A}$ \\
\hline
\end{tabular}

Note. $\widehat{\mathscr{R}}_{0 A}$ and $\widehat{\mathscr{R}}_{0}$ are from Table 10 .

TABLE 13: The value of $\mathscr{R}_{0 F}$ is related to $\widehat{\mathscr{R}}_{0 M F}, \widehat{\mathscr{R}}_{0}$, and $\widehat{\mathscr{R}}_{0 A}$ for the second scenario.

\begin{tabular}{|c|c|c|c|c|}
\hline The $n$ days at the i.t.o.p. & $\lambda$ & $\mathscr{R}_{0 F}$ & $\begin{array}{c}\widehat{\mathscr{R}}_{0 M F} \\
\text { for } k \in(0,2]\end{array}$ & Important notes \\
\hline II: 7 days & 0.019 & 1.35 & $(1.07,1.58]$ & $\mathscr{R}_{0 F} \in\left(\widehat{\mathscr{R}}_{0 M F} \cap \widehat{\mathscr{R}}_{0}\right) \backslash \widehat{\mathscr{R}}_{0 A}$ \\
\hline IV: 5 days & 0.048 & 1.83 & $(1.17,2.30]$ & $\mathscr{R}_{0 F} \in\left(\widehat{\mathscr{R}}_{0 M F} \cap \widehat{\mathscr{R}}_{0}\right) \backslash \widehat{\mathscr{R}}_{0 A}$ \\
\hline I: 5 days & 0.091 & 2.54 & $(1.32,3.35]$ & $\mathscr{R}_{0 F} \in\left(\widehat{\mathscr{R}}_{0 M F} \cap \widehat{\mathscr{R}}_{0}\right) \backslash \widehat{\mathscr{R}}_{0 A}$ \\
\hline V: 7 days & 0.105 & 2.76 & $(1.36,3.66]$ & $\mathscr{R}_{0 F} \in\left(\widehat{\mathscr{R}}_{0 M F} \cap \widehat{\mathscr{R}}_{0}\right) \backslash \widehat{\mathscr{R}}_{0 A}$ \\
\hline III: 6 days & 0.121 & 3.02 & $(1.40,4.03]$ & $\mathscr{R}_{0 F} \in\left(\widehat{\mathscr{R}}_{0 M F} \cap \widehat{\mathscr{R}}_{0}\right) \backslash \widehat{\mathscr{R}}_{0 A}$ \\
\hline
\end{tabular}

$\widehat{\mathscr{R}}_{0 A}$ and $\widehat{\mathscr{R}}_{0}$ are from Table 11 .

four persons during the time period of dengue infection. Meanwhile, the value of $\mathscr{R}_{0 M F}$ depends on the value of $k$. We show that for $k=1$, then $\mathscr{R}_{0 F}=\mathscr{R}_{0 M F}$ (values in bracket). The increases in the value of $\lambda$ and $k$ cause an increase in the value of $\mathscr{R}_{0 M F}$. While the value in the square brackets in Tables 5 and 7 shows the relationship between the value of $\lambda$ and $\rho$, so that $\mathscr{R}_{0 F}=\mathscr{R}_{0 A}$. We also found that an increase in the value of $\lambda$, for a particular value of $\rho$, does not always cause an increase in the value of $\mathscr{R}_{0 A}$. Particular information for the four t.o.p. in Tables 6 and 7 shows that, to obtain the four secondary infection cases from one primary infection, the number of mosquitoes should be at least twice as large as the human population, whereas in Tables 4 and 5 we do not obtain information about the ratio of the number of mosquitoes and humans. Therefore, $\mathscr{R}_{0 A}$ provides more complete information than $\mathscr{R}_{0 F}$ and $\mathscr{R}_{0 M F}$.

Next, From Tables 6 and 7, there is the value of $\rho$ such that $\mathscr{R}_{0 F}=\mathscr{R}_{0 A}$ for every dengue infection i.t.o.p. (see the value in brackets of Tables 6 and 7). For $k=1$, there are a certain pair of $\rho$ and $\lambda$ for every i.t.o.p., such that $\mathscr{R}_{0 A}=\mathscr{R}_{0 M F}=$ $\mathscr{R}_{0 F}$ (Table 4 ). For the first scenario, if $\rho$ is relatively small then the value of $\mathscr{R}_{0 \mathrm{~A}}$ is inversely proportional to the value of $\lambda$. For instance, if $\rho=0.67$ (see t.o.p. II, Table 6) then $\mathscr{R}_{0 A}$ increases or decreases with an increasing $\lambda$. The magnitude relation between $\lambda$ and $\mathscr{R}_{0}$ is not the case in other values of $\rho$. Also, we obtained that $\mathscr{R}_{0 F}$ is a special case of $\mathscr{R}_{0 A}$ for a certain pair of $\rho$ and $\lambda$. Thus, if we have the value of $\rho$ from the real-life situation then the value of $\mathscr{R}_{0 A}$ is more realistic than $\mathscr{R}_{0 F}$ when estimating $\mathscr{R}_{0}$ from the real-life dengue epidemic.

Next, in the first scenario, the value of $\mathscr{R}_{0 F}$ is not only contained in $\widehat{\mathscr{R}}_{0 M F}$ and $\widehat{\mathscr{R}}_{0 A}$ but also contained within $\widehat{\mathscr{R}}_{0}$. Also, the value of $\mathscr{R}_{0 F}$ belongs to the intersection of $\widehat{\mathscr{R}}_{0 M F}$ and $\widehat{\mathscr{R}}_{0 A}$. This is also the case in the first scenario, where $\mathscr{R}_{0 F}$ have good precision but not as good a predictor when compared with $\widehat{\mathscr{R}}_{0 M F}$ and $\widehat{\mathscr{R}}_{0 A}$. Whilst $\widehat{\mathscr{R}}_{0 M F}$ and $\widehat{\mathscr{R}}_{0 A}$ are good predictors, they do not have a good precision (see Tables 7-13). In the second scenario, the value of $\widehat{\mathscr{R}}_{0 F}$ is contained within the range of $\widehat{\mathscr{R}}_{0 M F}$ and $\widehat{\mathscr{R}}_{0}$ values but not contained in $\widehat{\mathscr{R}}_{0 A}$ range. Furthermore, the value of $\mathscr{R}_{0 F}$ is lower than range's lower end of values of $\widehat{\mathscr{R}}_{0 A}$ (see Tables $10-13)$. Meanwhile, it is not enough just to pay attention to $\widehat{\mathscr{R}}_{0}$ value of the model in (1). Therefore, from the aspect of the efficiency and effectiveness of dengue control, the government should pay attention to the basic reproductive ratio estimation of $\mathscr{R}_{0 A}$ that is reported here, in order to improve the quality control of the spread of dengue fever.

The important notes in Tables $10-13$ show that $\mathscr{R}_{0 F}$ value (see Table 4 or Tables $5-7$ ) is included in the intervals $\widehat{\mathscr{R}}_{0 M F}$, $\widehat{\mathscr{R}}_{0 A}$, and $\widehat{\mathscr{R}}_{0}$ for the first scenario. It means that the value of $\mathscr{R}_{0 F}$ can still be used as a standard for the control of dengue virus infection. However, to obtain the maximum value of $\mathscr{R}_{0 A}=2-12$, then the number of mosquitoes would be 2-8 times greater than the number of people, with the takeoff rate of 0.121; see Table 11. Therefore, the use of value of $\rho$, $\lambda$, and $\mathscr{R}_{0 A}$ as a standard value for the control of a dengue endemicity is more realistic than the others.

From Figures 7-14, not only do we obtain the value of the basic reproductive ratio estimation of the $\mathscr{R}_{0 A}$ which is greater than one but also we do obtain the value which is less than one; for instance, from the first scenario at $\lambda=$ 0.190 and $\rho=0.67$ we have $\mathscr{R}_{0 A}=0.67<1$ (see Table 7). Also, we can determine the intersection range of a basic reproductive ratio from a different construction of value of $\lambda$. Both proposed constructions of $\mathscr{R}_{0}$ are the same when using assumption in [12] for these particular cases. But increasing the number of secondary cases for every t.o.p. will depend on $k$ for the first construction and depend on $\rho$ for the second construction. These results indicate that 


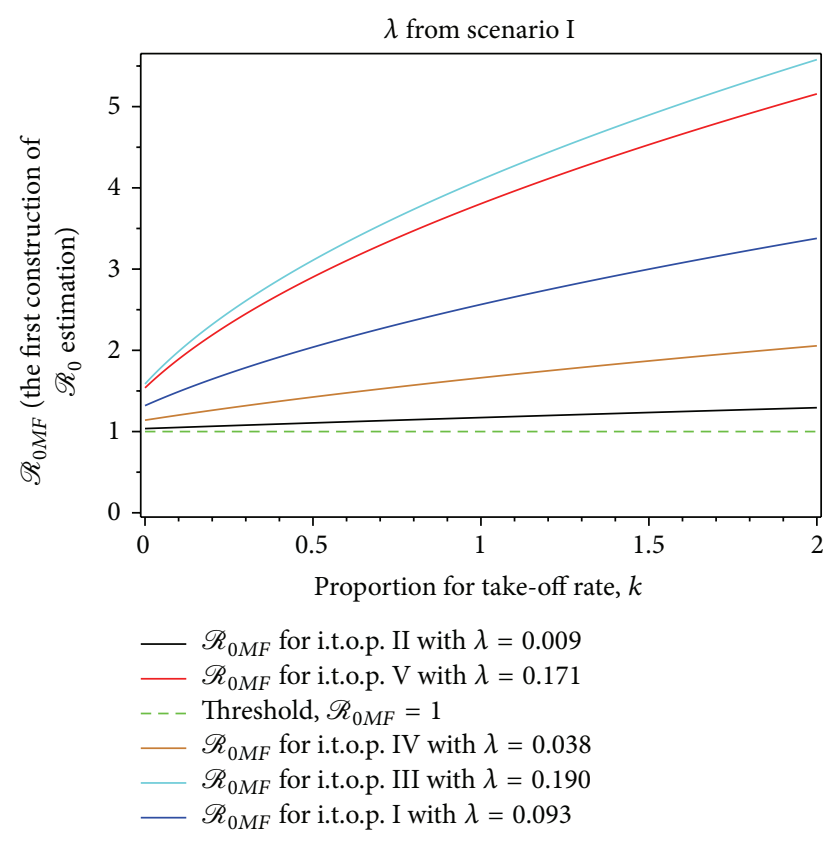

Figure 7: The dynamic of $\mathscr{R}_{0 M F}$ for the dengue outbreak at the beginning of the t.o.p. as a function of the infected mosquito per human index for $\lambda$ from the first scenario.

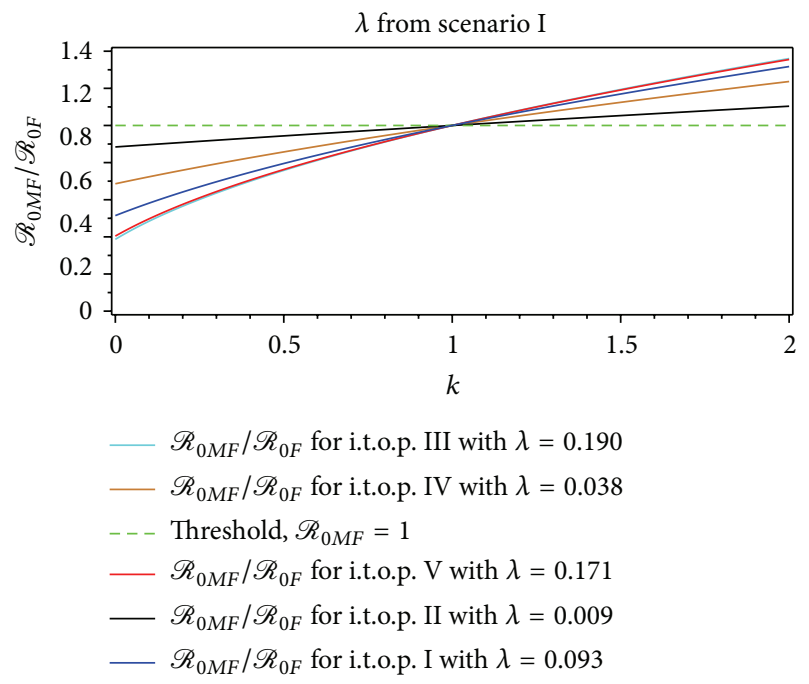

Figure 8: The ratio of $\mathscr{R}_{0 M F} / \mathscr{R}_{0 F}$ for the dengue outbreak at the beginning of the t.o.p. as a function of the ratio of the rate of the infected mosquito per human index $k$ for $\lambda$ from the first scenario.

the greater the number of mosquitoes means the higher the number of new cases of dengue and the shorter the period of the i.t.o.p. of dengue infection. Furthermore, $\mathscr{R}_{0 F}$ estimates of $\mathscr{R}_{0}$ are too low when compared to $\mathscr{R}_{0 A}$. This is very dangerous in application, since the intervention to control dengue that results from $\mathscr{R}_{0 F}$ estimation will not be able to stop the disease once it exists. Therefore, prevention of dengue incidences is more appropriate if it is done early, and exactly how early is dependent on $\mathscr{R}_{0 A}$ estimation.

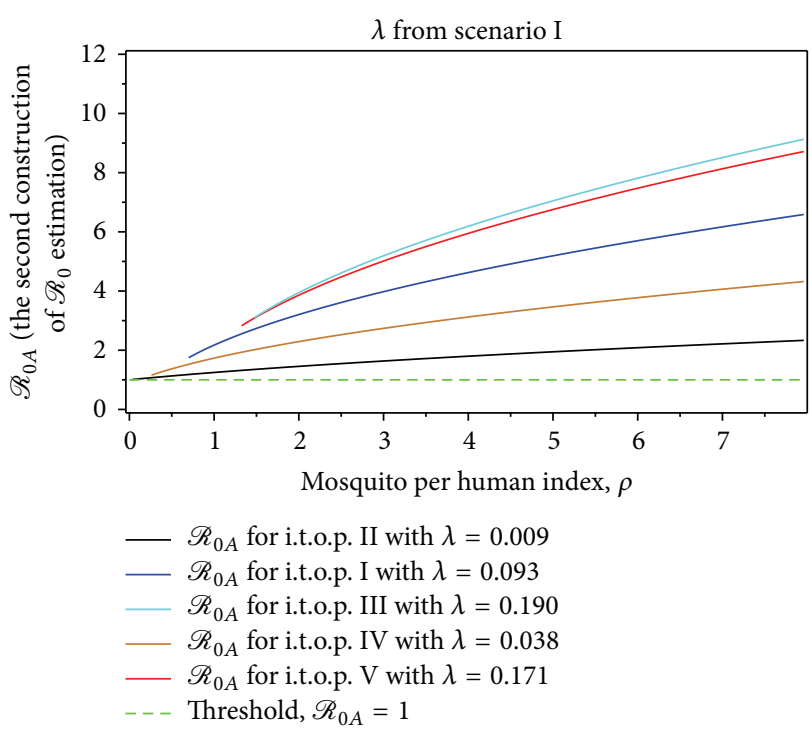

Figure 9: The dynamic of $\mathscr{R}_{0 A}$ for the dengue outbreak at the beginning of the t.o.p. as a function of the mosquito per person index for $\lambda$ from the first scenario.

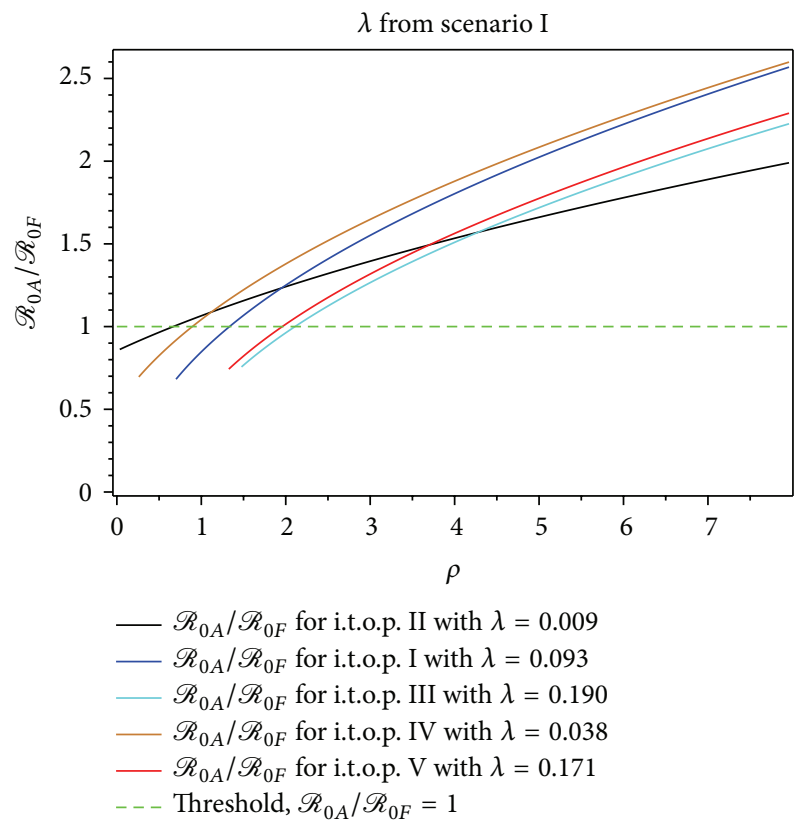

Figure 10: The ratio of $\mathscr{R}_{0 A} / \mathscr{R}_{0 F}$ for the dengue outbreak at the beginning of the t.o.p. as a function of the infected mosquito per human index $\rho$ for $\lambda$ from the first scenario.

We used real dengue epidemic data from a dengue epidemic, between the dates Nov. 25, 2008, and 2012 from SB Hospital. This includes the estimation of the basic reproductive ratio at the beginning of t.o.p. of dengue infection by using $\mathscr{R}_{0 F}, \mathscr{R}_{0 M F}$, and $\mathscr{R}_{0 A}$ for $\mathscr{R}_{0}$ estimation. $\mathscr{R}_{0}$ magnitude estimation under the same assumption was conducted in [12], resulting in $\mathscr{R}_{0 F}$. We propose $\mathscr{R}_{0 M F}$ and $\mathscr{R}_{0 A}$ for comparing with $\mathscr{R}_{0 F}$ for $\mathscr{R}_{0}$ estimation from dengue incidence data from SB Hospital. As a results, we get $\mathscr{R}_{0 M F}>1.59$ from the first 


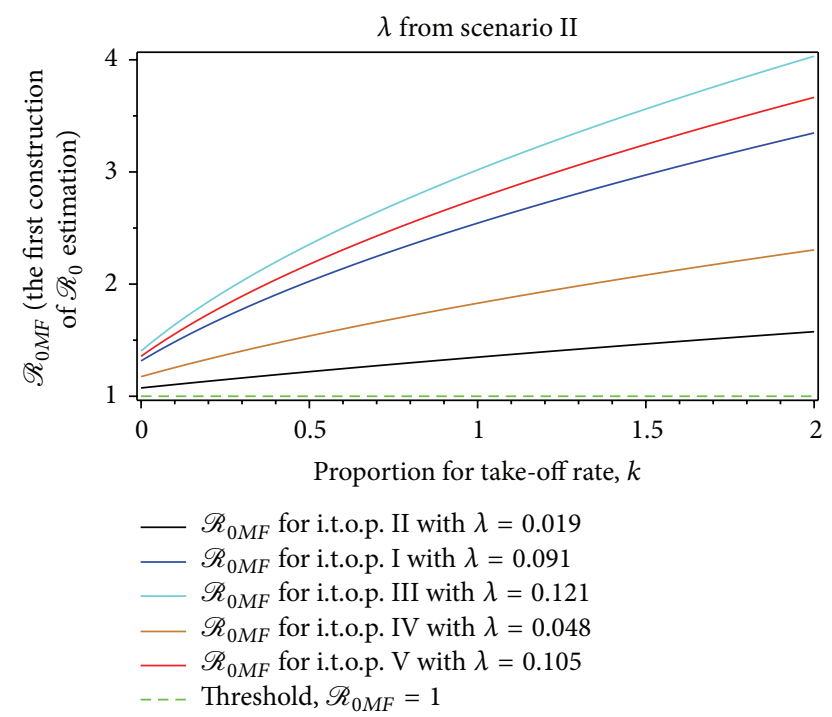

Figure 11: The dynamic of $\mathscr{R}_{0 M F}$ for the dengue outbreak at the beginning of the t.o.p. as a function of the infected mosquito per human index for $\lambda$ from the first Scenario.

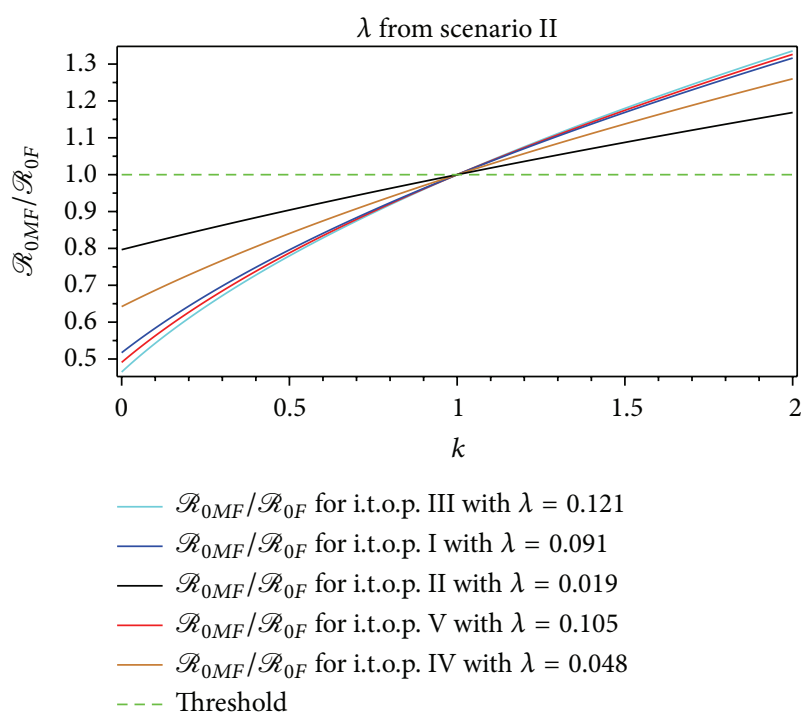

FIGURE 12: The ratio of $\mathscr{R}_{0 M F} / \mathscr{R}_{0 F}$ for the dengue outbreak at the beginning of the t.o.p. as a function of ratio of the rate of the infected mosquito per human index $k$ for $\lambda$ from the second scenario.

scenario (Table 4, Figures 7 and 9) and $\mathscr{R}_{0 M F}>1.40$ from the second scenario, respectively (Table 6, Figures 7 and 9). From the first scenario, we obtained the the basic reproductive ratio was lowest in the t.o.p. II $[1.01,2.33)$ and largest in the t.o.p. III $[3 \cdot 10,9.13)$ (Table 10, Figures 8 and 10). Furthermore, from the second scenario, the basic reproductive ratio was lowest in the t.o.p. II [2 $37,7.93)$ and largest in the t.o.p. III $[5.48,11.78)$ (Table 11, Figures 8 and 10). We believe that $\mathscr{R}_{0 M F}$ and $\mathscr{R}_{0 A}$ are a good $\mathscr{R}_{0}$ estimation at the beginning of the take-off period of infection. Thus, our results can be considered in the control of a dengue fever epidemic, in the dengue endemic regions of Bandung, Indonesia. But the effects of dengue

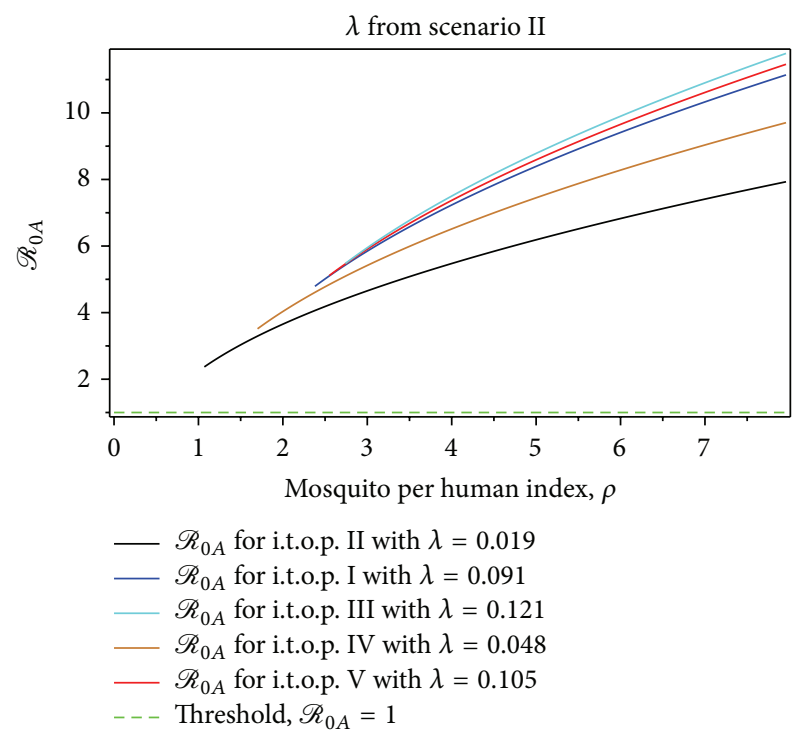

FIgURE 13: The dynamic of $\mathscr{R}_{0 A}$ for the dengue outbreak at the beginning of the t.o.p. as a function of the mosquito per person index for $\lambda$ from the second scenario.

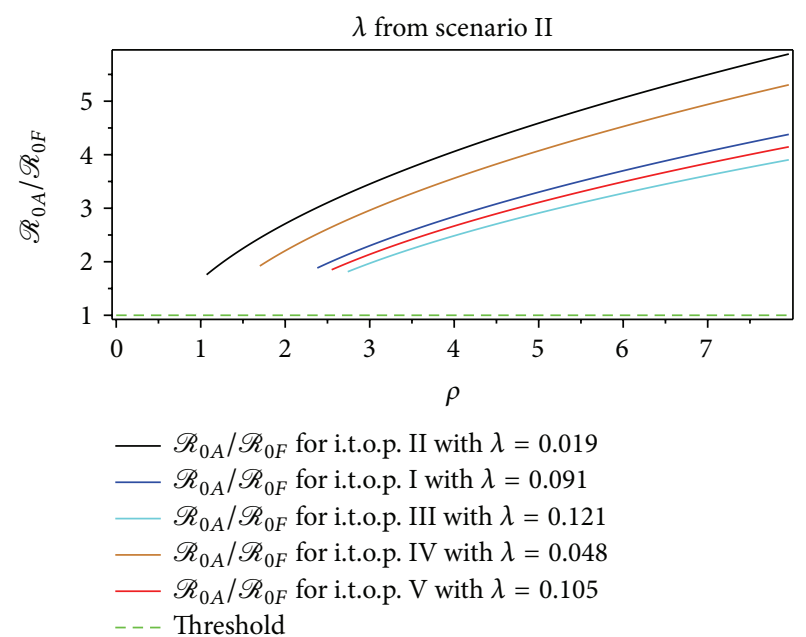

FIgURE 14: The ratio of $\mathscr{R}_{0 A} / \mathscr{R}_{0 F}$ for the dengue outbreak at the beginning of the t.o.p. as a function of the infected mosquito per human index $\rho$ for $\lambda$ from the second scenario.

incidence data were obtained from SB Hospital, Bandung, to the value of $\mathscr{R}_{0}$ estimation, and describe the transmission of dengue fever, because only the dengue incidence data of the same serotype were considered.

Some of the results of the basic reproductive ratio estimation for dengue fever in compartmental model were reported in several publications. Favier et al. in [12] estimated a basic reproductive ratio, which ranged widely from 2.0 to 103 for dengue epidemics occurring during the years 1996 to 2003 in 9 Brazilian regions. The upper bound of their range, in estimating the basic reproductive ratio, is significantly higher than the upper bound estimate reported here. The lower bound of their range estimate, of basic reproductive 
ratio, is relatively smaller than the lower bound estimate reported here for some take-off period but the others are relatively higher than the lower bound estimate reported here (Tables 4 and 6). Chowell et al. in [11] estimated the basic reproductive ratio, by using the relationship between the basic reproductive ratio and the growth rate in the early phase of dengue fever transmission in Toceman, Mexico. Their mean estimate of the basic reproductive ratio is 4.22 , which is significantly higher than the mean estimate reported here for every take-off period which is obtained by $\mathscr{R}_{0 F}$. The weekly number of dengue cases in Peru for the period 1994-2006 was analyzed in [21] which obtained that the basic reproductive ratio had a median $1.76(0.83-4.46)$, where these values inside the value of $\mathscr{R}_{0}$ are reported here; see Table 7 . During the 2000 epidemic, occurring in 12 cities of the state of Sao Paulo, Brazil, [4] estimated that the basic reproductive ratio was in the range [3.6, 12.9]. More recently [14] estimated the reproductive ratio by using the relationship between the basic reproductive ratio and the force of infection of dengue fever transmission in Salvador from periods 1996 to 1997, and 2002. They obtain an estimation of $\mathscr{R}_{0}=2.85(2.77,3.70)$ in the periods 1996 to 1997 , and $\mathscr{R}_{0}=2.65(2.50,3.30)$ for 2002. The newest report of $\mathscr{R}_{0}$ estimation is in [22] for dengue epidemics in 2002 dengue outbreak in Easter Island, Chile. Their mean of the basic reproductive ratio $\mathscr{R}_{0}$ at 27.2 is significantly higher than every value of $\mathscr{R}_{0}$ estimation reported here for five initial take-off periods of dengue infection from two scenarios for estimating of $\lambda$. Our estimation results, for the basic reproductive ratio by using $\mathscr{R}_{0 F}$ range widely, are from 1.17 to 3.89 for the first scenario and from 1.35 to 3.02 for the second scenario which belongs to the range of the first scenario. Our $\mathscr{R}_{0 M F}$ estimation accommodates for the difference of the take-off rate $\lambda$ between mosquito and human. If there are no differences in the take-off rate then [12] estimates are exactly the same as ours. Otherwise $\mathscr{R}_{0 F}$ could overestimate or underestimate depending on the value of $k$. Tables 4 and 6 show that $\mathscr{R}_{0 A}$ accommodates differences in $\rho . \mathscr{R}_{0 F}$ is exactly the same as our second estimates for a certain pair of $\rho$ and $\lambda$. Otherwise $\mathscr{R}_{0 F}$ could overestimate or underestimate depending on the value of $\rho$, and so forth. Therefore, our $\mathscr{R}_{0 M F}$ and $\mathscr{R}_{0 A}$ constructions are more realistic for estimating the value of the basic reproductive ratio, than the $\mathscr{R}_{0 F}$.

In the first scenario, the control of dengue fever, which is based on the standard value $\mathscr{R}_{0 F}$, is still realistic. Furthermore, better control of dengue fever, when based on the maximum value of $\mathscr{R}_{0 M F}$ or maximum value of $\mathscr{R}_{0 A}$, is shown in Table 6 and Figures 7-10. While for the second scenario, which is shown by Table 6 and Figures 11-14, it is better to choose one value from the interval as a standard value used to control dengue fever. Therefore, the second construction is better choice, for dengue control strategies in Bandung, Indonesia. Nevertheless, the estimation of $\mathscr{R}_{0}$ has limitations, because it is only done from the fourth day until the seventh day, so the effect of the increase in the incidence of dengue $\mathscr{R}_{0}$ is no longer considered. Furthermore, the dengue incidence data used, from SB Hospital, do not distinguish between the latent population and the dengue infected human population, so the estimate of $\mathscr{R}_{0}$ is limited by the data characteristics. In reality, nobody can distinguish between the latent population and the dengue infected population. Furthermore the latent population has a short incubation time period.

\section{Conclusion}

We have conducted two different constructions of basic reproductive ratio estimations based on incidence data. These constructions modify $\mathscr{R}_{0 F}$ model by allowing the take-off rates of human and mosquito to vary. In the first construction, we assumed that the take-off rate of an infected mosquito is proportional to the take-off rate of a human. This construction leads to a new form of modified basic reproductive ratio estimation for $\mathscr{R}_{0 M F}$ was a function of the take-off rate of a human $(\lambda)$ and the take-off rate of a mosquito $(k \lambda)$. A sensitivity analysis indicated that a small variation of a mosquito take-off rate could affect significant change in the basic reproductive ratio. In the second construction we assumed that, during the take-off period, the initial growth of infected humans is slightly affected by the growth of infected mosquitoes, and vice versa. This construction leads to a one-parameter coupling of the initial growth of infected human and mosquitoes. The modified basic reproductive ratio estimation for $\mathscr{R}_{0 A}$ resulted from this construction, and it depends on the mosquitoes per person index $\rho$. This ratio increases for small take-off rate and decreases for larger takeoff rate. A numerical analysis of $\mathscr{R}_{0 M F} / \mathscr{R}_{0 F}$ and $\mathscr{R}_{0 A} / \mathscr{R}_{0 F}$ ratios shows variation in a relatively large range of values for $\mathscr{R}_{0 F}$, in respect to $(\lambda, k)$ and $(\lambda, \rho)$ for the first and second scenario for determined $\lambda$, respectively.

\section{Conflict of Interests}

The authors declare that there is no conflict of interests regarding the publication of this paper.

\section{Acknowledgments}

This work was supported by the Hibah Stretegis Nasional, under the DIKTI Indonesia scheme. The authors would like to thank to anonymous reviewers for their very helpful suggestions and comments that helped to improve the paper.

\section{References}

[1] M. N. Burattini, M. Chen, A. Chow et al., "Modelling the control strategies against dengue in Singapore," Epidemiology and Infection, vol. 136, no. 3, pp. 309-319, 2008.

[2] WHO, "Dengue guidelines for diagnosis, treatment, prevention and control 2012," New Edition Sheet, World Health Organization, Geneva, Switzerland, 2012, http://www.who.int/denguecontrol/resources/en/.

[3] C. A. Marques, O. P. Forattini, and E. Massad, "The basic reproduction number for dengue fever," Transactions of the Royal Society of Tropical Medicine and Hygiene, vol. 10, no. 8, p. 5859, 1994.

[4] E. Massad, F. A. B. Coutinho, M. N. Burattini, and L. F. Lopez, "The risk of yellow fever in a dengue-infested area," Transactions 
of the Royal Society of Tropical Medicine and Hygiene, vol. 95, no. 4, pp. 370-374, 2001.

[5] E.-E. Ooi, K.-T. Goh, and D. J. Gubler, "Dengue prevention and 35 years of vector control in Singapore," Emerging Infectious Diseases, vol. 12, no. 6, pp. 887-893, 2006.

[6] L. Esteva and C. Vargas, "Analysis of a dengue disease transmission model," Mathematical Biosciences, vol. 150, no. 2, pp. 131151, 1998.

[7] O. Diekmann and J. A. P. Heesterbeek, Mathematical Epidemiologi of Infectious Diseases: Model Building, Analisis and Interpretation, Wiley Series in Mathematical and Computational Biology, Editor in chief Simon Levin, Princeton University, Princeton, NJ, USA, 2000.

[8] P. van den Driessche and J. Watmough, "Reproduction numbers and sub-threshold endemic equilibria for compartmental models of disease transmission," Mathematical Biosciences, vol. 180, pp. 29-48, 2002.

[9] J. M. Haffernan, R. J. Smith, and L. M. Wahl, "Perspectives on the reproductive ratio," Journal of The Royal Society Interface, vol. 2, pp. 281-293, 2005.

[10] C. C. Chaves, Z. Feng, and W. Huang, "On computation of $R_{0}$ and its rule on global stability," 2009, https://ecommons.cornell .edu/bitstream/1813/32146/1/BU-1553-M.pdf.

[11] G. Chowell, P. D. Dueñas, J. C. Miller et al., "Estimation of the reproduction number of dengue fever from spatial epidemic data," Mathematical Biosciences, vol. 208, no. 2, pp. 571-589, 2007.

[12] C. Favier, N. Degallier, M. G. Rosa-Freitas et al., "Early determination of the reproductive number for vector-borne diseases: the case of dengue in Brazil," Tropical Medicine and International Health, vol. 11, no. 3, pp. 332-340, 2006.

[13] A. K. Supriatna, "Estimating the basic reproduction number of dengue transmission during 2002-2007 outbreaks in Bandung, Indonesia," Dengue Bulletin, vol. 33, no. 1, pp. 21-32, 2009.

[14] S. T. R. Pinho, C. P. Ferreira, L. Esteva, F. R. Barreto, V. C. M. e Silva, and M. G. L. Teixeira, "Modelling the dynamics of dengue real epidemics," Philosophical Transactions of the Royal Society A: Mathematical, Physical and Engineering Sciences, vol. 368, no. 1933, pp. 5679-5693, 2010.

[15] M. Amaku, F. Azevedoa, M. N. Burattini, F. A. B. Coutinho, L. F. Lopez, and E. Massad, "Interpretations and pitfalls in modelling vector-transmitted infections," Epidemiology and Infection, vol. 9, pp. 1803-1815, 2014.

[16] G. Macdonald, “The analysis of equilibrium in malaria," Tropical Diseases Bulletin, vol. 49, no. 9, pp. 813-829, 1952.

[17] L. Esteva and C. Vargas, "A model for dengue disease with variable human population," Journal of Mathematical Biology, vol. 38, no. 3, pp. 220-240, 1999.

[18] N. Nuraini, E. Soewono, and K. A. Sidarta, "Mathematical model of dengue disease transmission with severe DHF conpartment," Bulletin of the Malaysian Mathematical Sciences Society, vol. 30, no. 2, pp. 143-157, 2007.

[19] P. Pongsumpun and I. M. Tang, "A realistic age structured transmission model for dengue hemorrhagic fever in Thailand," Southeast Asian Journal of Tropical Medicine and Public Health, vol. 32, no. 2, pp. 336-340, 2001.

[20] O. Diekmann, J. A. P. Heesterbeek, and J. A. J. Metz, "On the definition and the computation of the basic reproduction ratio $R_{0}$ in models for infectious diseases in heterogeneous populations," Journal of Mathematical Biology, vol. 28, no. 4, pp. 365-382, 1990.
[21] G. Chowell, C. A. Torre, C. Munayco-Escate et al., "Spatial and temporal dynamics of dengue fever in Peru: 1994-2006," Epidemiology and Infection, vol. 136, no. 12, pp. 1667-1677, 2008.

[22] G. Chowell, R. Fuentes, A. Olea, X. Aguilera, H. Nesse, and J. M. Hyman, "The basic reproduction number $R_{0}$ and effectiveness of reactive interventions during dengue epidemics: the 2002 dengue outbreak in Easter Island, Chile," Mathematical Biosciences and Engineering, vol. 10, no. 5-6, pp. 1155-1174, 2013. 


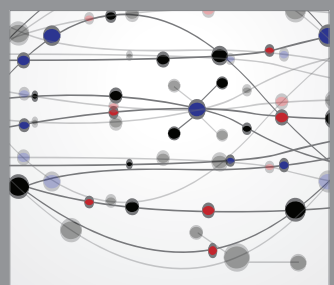

The Scientific World Journal
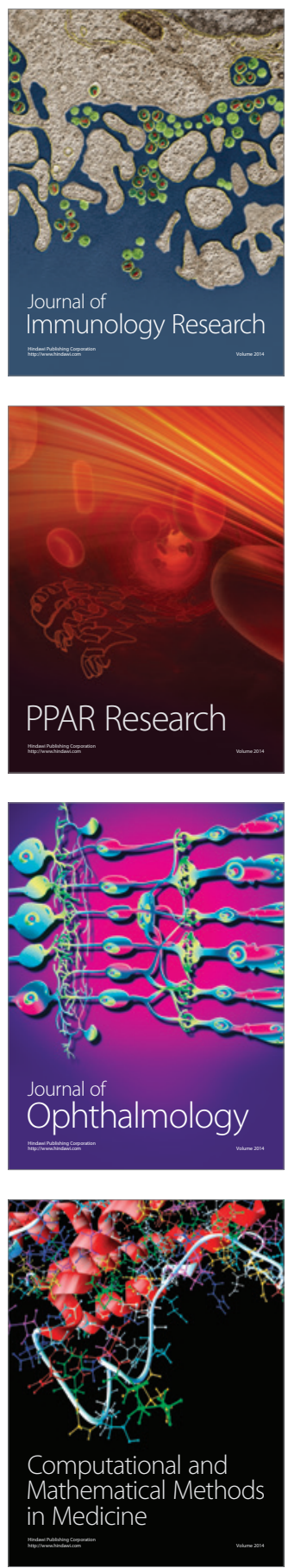

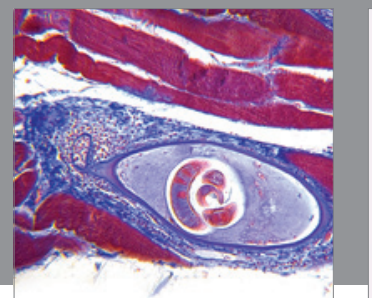

Gastroenterology

Research and Practice
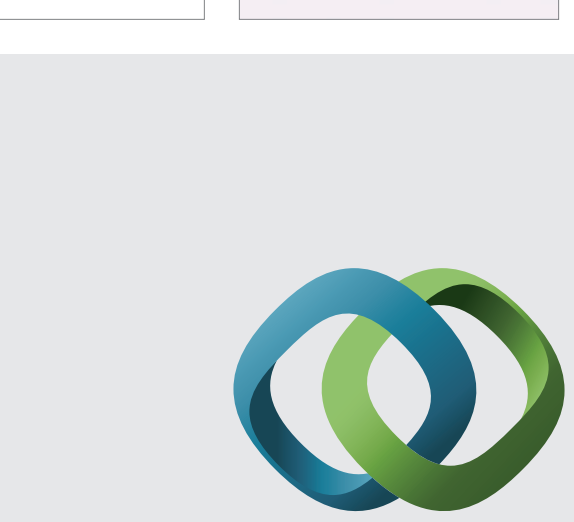

\section{Hindawi}

Submit your manuscripts at

http://www.hindawi.com
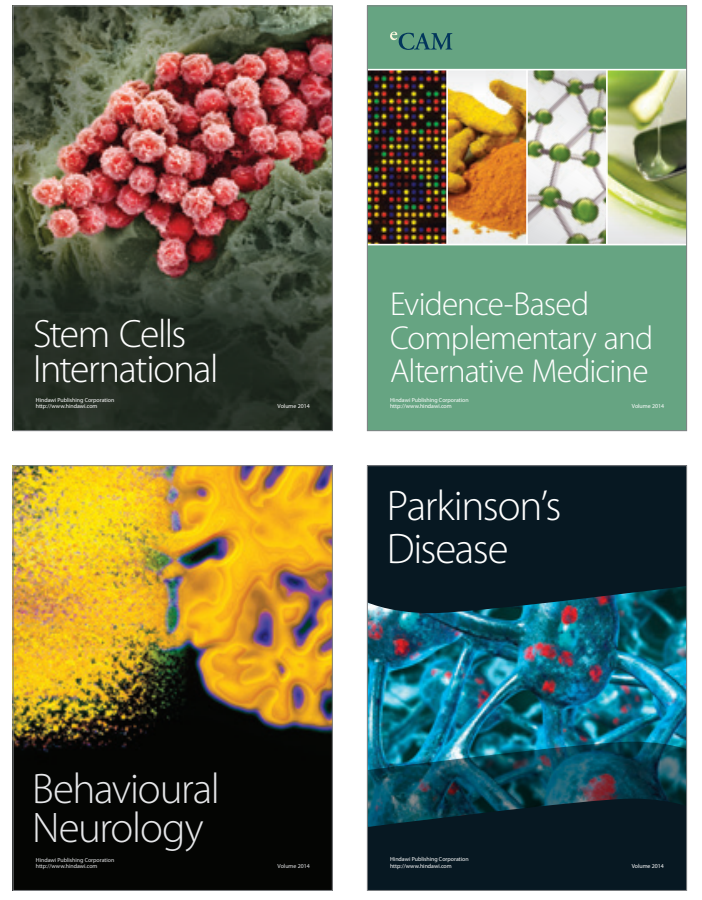
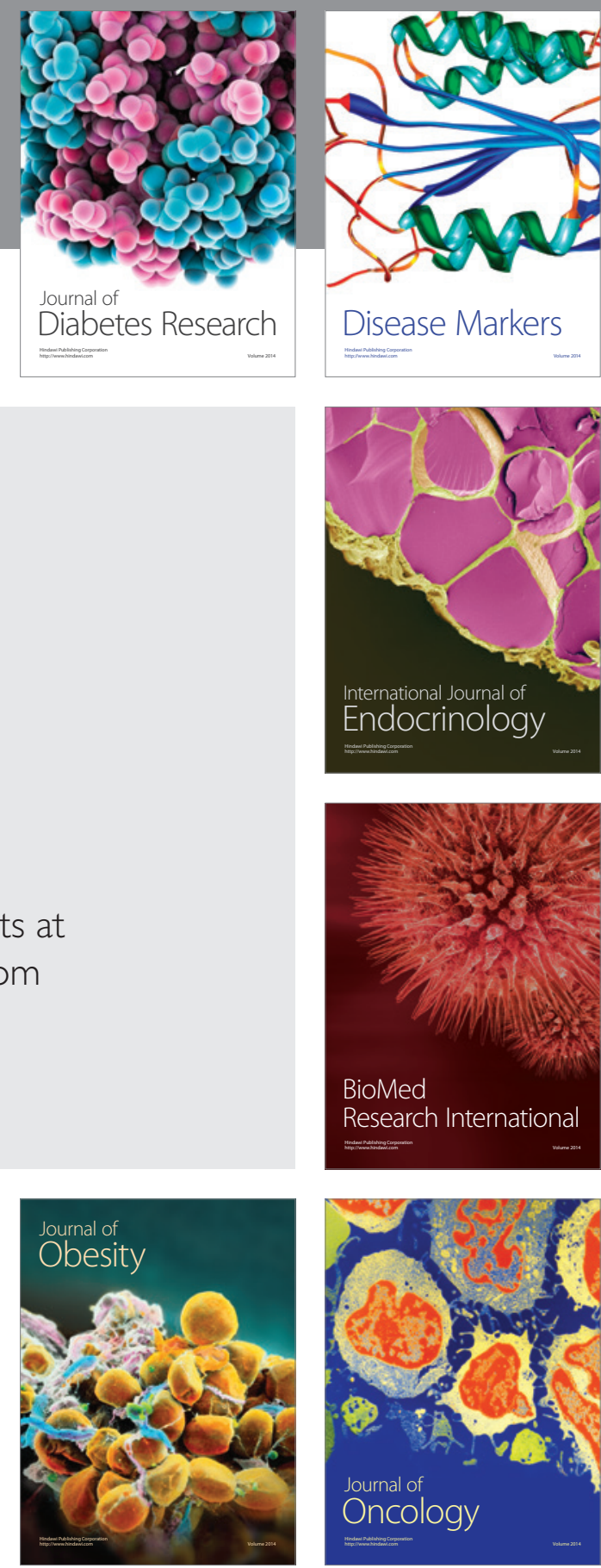

Disease Markers
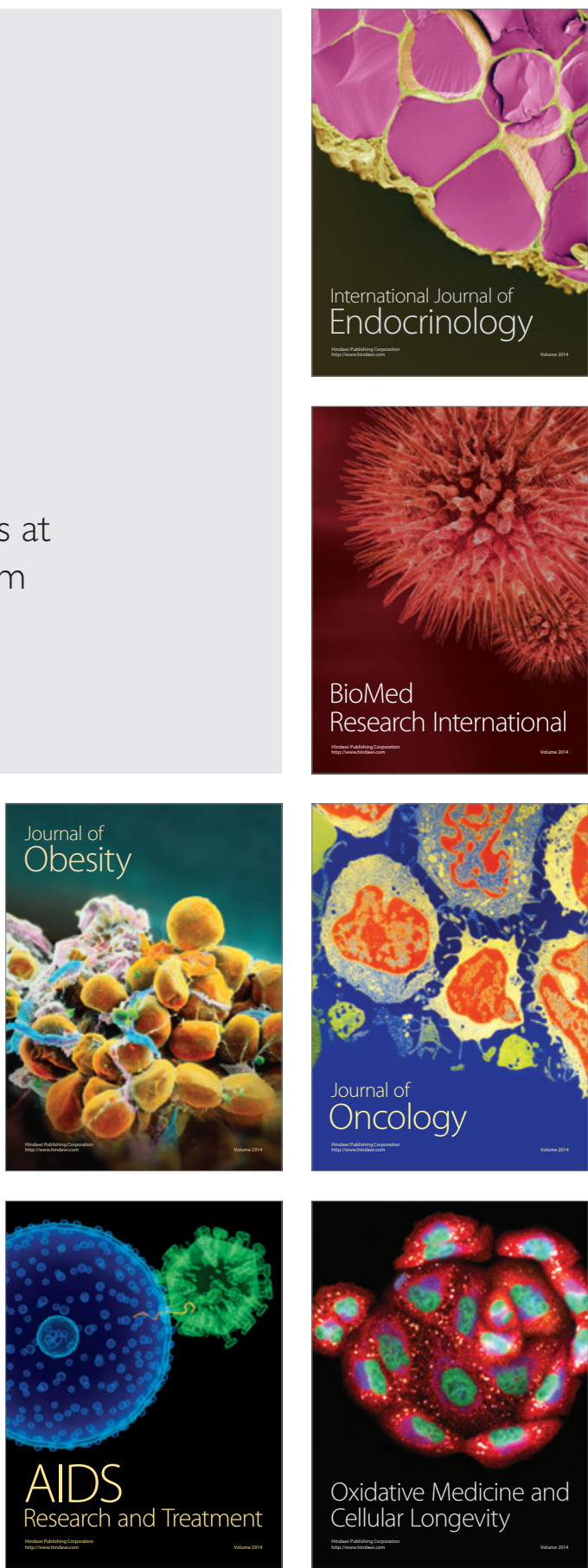